Sharif University of Technology
Scientia Iranica
SCIENTIA $\quad \begin{gathered}\text { Transactions D: Computer Science \&s Engineering and Electrical Engineering } \\ \text { http://scientiairanica.sharif.edu }\end{gathered}$

\title{
Heat and electricity supply chain expansion planning under the umbrella of energy hub: A case study of Iran
}

\author{
H. Sadeghi ${ }^{a}$, M. Rashidinejad ${ }^{\mathrm{a}}$, M. Moeini-Aghtaie ${ }^{\mathrm{b}}$, and A. Abdollahi ${ }^{\mathrm{a}, *}$ \\ a. Department of Electrical Engineering, Shahid Bahonar University, Kerman, P.O. Box 76169-133, Iran. \\ b. Department of Electrical Engineering, Sharif University of Technology, Tehran, P.O. Box 11365-11155, Iran. \\ Received 23 January 2020; received in revised form 29 May 2020; accepted 9 November 2020
}

\author{
KEYWORDS \\ Energy hub; \\ Heat; \\ Gas and electricity \\ supply chains; \\ Co-expansion \\ planning; \\ Gas storage systems.
}

\begin{abstract}
Reinforcing the correlation between Gas and Electricity Systems (G\&ESs) susceptible to diverse factors ranging from anthropogenic climate change to the advent of new conversion/generation technologies has remarkably brought into focus the co-expansion of G\&ESs using a new concept so-called Energy Hub (EH) as well as the potential of storage systems. To assess the effectiveness of EH approach and the role of storages in the coordinated plans of G\&ES, this study proposes a comprehensive EH-based planning model for co-expansion of G\&ES supply chains with respect to the role of Gas Storage Systems (GSSs). As a Mixed-Integer Linear Programming (MILP) problem, the model is applied to a real large-scale case study, i.e., the Iranian G\&ES, and is solved via GAMS package. Simulation results reveal that incorporation of the interactions existing between G\&ESs into their planning problems in the framework of an EH can reach more flexible, realistic, and optimal expansion plans than their traditional integrated expansion planning methods. Furthermore, findings indicate that the involvement capacities of GSSs provide an opportunity for optimal matching of demand with supply by increasing the productivity of the gas pipelines, thus allowing technically and economically sensible longterm management of gas supply systems.
\end{abstract}

(C) 2022 Sharif University of Technology. All rights reserved.

\section{Introduction}

Being one of the biggest sources of $\mathrm{CO}_{2}$ emission, power plants play a prominent role in the realization of green economy through the modification of their own operation and expansion strategies. The need to apply emission restrictions to the generation sectors has propelled different countries to use energy sources with lower emission. Accordingly, Natural Gas (NG) will have the main role among fossil fuels in the future of conventional Energy Systems (ESs) [1].

*. Corresponding author. Tel.: +983433202519 E-mail address: a.abdollahi@uk.ac.ir (A. Abdollahi)

doi: $10.24200 /$ sci. 2020.55301 .4151
However, the rotation of visions to the NG energy is also derived from climate change. In this context, the need for increasing the capacity of fast-response generation units in parallel with faster penetration of Renewable Energy Sources (RES), as non-dispatchable and inherently uncertain energy sources, and lower NG price than other fossil fuels are, in fact, comprised of other well-known factors that strengthen the position of NG in the future of energy paradigm. Redundancy of NG natural sources, advances in extraction of NG from unconventional sources in terms of shale gas, and economic features of Gas-fired Power Plants (GPG), such as lower investment cost as well as quicker installation, have also had effective roles in enhancing NG position.

All the above-enumerated factors along with in- 
tent of some developed countries like Germany and Switzerland to accelerate the processes of retiring coal-fired power plants, replacing them with gas-fired ones, and putting forward nuclear generation phaseout programs in the wake of Fukushima disaster in 2011 have significantly raised the interdependency of Gas and Electricity Systems (G\&ESs) $[2,3]$. In doing so, the role of technological progresses in the field of distribution of energy resources for microgrids $[4,5]$ as well as the advent of diverse energy converters in intensifying this interdependency is undeniable. Gas-fired Distributed Generations (GDG), Power to Gas (P2G) storage systems, heat pumps (HPs) and, ultimately, Combined Heat and Power (CHP) units are some of those technologies that have recently had a fast penetration rate in ESs as a result of global concentrations on Energy Efficiency (EE) and clean energy production improvements.

Obviously, widespread penetration of NG-based energy conversion/generation technologies in the generation mix of power and heating systems impresses generation, transmission, and distribution of the most common energy demand forms, i.e., heat and electrical energies. This needs to employ more comprehensive methodologies to plan and analyze today's ESs in an integrated manner in terms of multi-energy system to facilitate green economic realization. This newly emerged viewpoint on ESs, nowadays, is pursued in a framework, so called "Energy Hub" (EH), which provides an opportunity to achieve more optimal and realistic operation and expansion strategies from all technical, economical, and environmental perspectives [6].

\subsection{Literature review}

What makes the utilization of EH concept essential to Energy Systems' Expansion Planning (ESEP) problems is the increased dependency of ESs on each other. The necessity of devoting greater attention to the application of $\mathrm{EH}$ approach is justified by the incremental rate of demand growth. In addition, (a) ES requirements including higher EE, better asset management, and more precise planning models owing to tremendous capability enhancement of computer systems and (b) soft computing methods realizing the optimization of sophisticated models add to the abovementioned justification. A review of the advantages and capabilities originating from this approach to ESs planning, scrutinized by a multitude of studies [3-13], helps appreciate this concept better.

With or without considering the enumerated factors associated with increase in today's interdependencies of ESs, up to now, the amalgamated expansion planning of G\&ES has been addressed by a wide range of research studies. Accuracy and method of modeling, employed optimization methodologies, and infrastructures considered in expansion planning make a distinction among research studies. In the case of hourly fluctuation in gas and electricity demands in an 11-year horizon, a precise linear dynamic model for expansion planning of Brazilian G\&ES was presented in [14]. In an almost similar framework, Barati et al. addressed simultaneous expansion planning of G\&ES on a large-scale and 7-year horizon [15]. The results obtained from conducting scenarios on the Iranian G\&ES illustrate the effectiveness of integrated expansion planning approach compared with separate planning of these systems. In [16], optimal expansion strategies of integrated gas and electricity distribution networks were determined and similarly, the findings indicated that simultaneous planning of the networks could remarkably reduce both operation and investment expenditures of both systems which, in turn, attenuates the social welfare. With emphasis on the importance of $\mathrm{NG}$ role in mitigating the share of generation sector in Greenhouse Gas (GHG) emission, Qui et al. suggested a suitable mixed-integer linear model for integrated expansion planning of G\&ES with respect to the combination of relevant energy markets in [17]. Similar to the results of the frameworks built in [18] and [19] that explored optimal capacity expansion strategies of GPG units integrated with gas and electricity transmission networks in the presence of uncertainties in relevant markets, simulation results of the current work enumerate the advantages of integrating G\&ES expansion planning problems in four general cases. The findings, first, demonstrate that the planning integration leads to the reduction of capital and operation costs as well as social welfare enhancement. The second and third advantages include (a) better identification of infrastructures weakness to meet energy demands on a long-term horizon and (b) better appreciation of the interactions between G\&ES to achieve a better coordination for relevant decision-making systems. Finally, the authors conclude that integration of G\&ES expansion planning problems enhances EE and asset management. Evaluation of the impacts of NG network constraints on co-optimization expansion planning of power generation and transmission systems [20] and simultaneous expansion planning of gas and electricity transmission networks taking into consideration the interaction between wind and fastresponse GPG units [21] are other pursued linchpins in the field of integrated expansion planning of ESs. Upon reviewing the above-mentioned research studies, none of them, hereafter called conventional integrated ESEP studies, distinguishes thermal load from total NG demand and considers the capability to convert energy forms into each other to meet end-user demanded energy forms, whereas the latter is the basis of $\mathrm{EH}$ approach [22].

Despite the obvious role of $\mathrm{EH}$ approach in 
achieving more optimal planning strategies, only a few research works in the ESEP field have focused on investigating the effectiveness of EH concept in coexpansion of G\&ES. The presented framework in [7], attempting to determine optimal expansion strategies of distribution networks, is indeed one of the first EH-based ESEP studies. However, the role of EH approach in the long-term co-expansion of G\&ES, for the first time, was scrutinized in [8]. This study indeed presented a linear and simplified model that only considered the main infrastructures and converters of the ESs, i.e., power generation capacity, gas and electricity transmission corridors, CHP units, and gas furnaces, to meet forecasted thermal and electrical loads at minimum cost. The simulated scenarios demonstrate that planning under the umbrella of $\mathrm{EH}$ faces a certain degree of flexibility for energy supply, resulting in lower investment and operation costs, higher reliability, and higher EE. Moreover, authors in [9-13] addressed operation planning of heat, gas, and electricity systems in the framework of an $\mathrm{EH}$.

To explain the energy supply flexibility from the viewpoint of EH, the effectiveness of Gas Storage Systems (GSSs) has not received enough attention until now so that only a few recently published articles have incorporated the impact of GSSs into the planning models. In this regard, Wang et al. proposed a bilevel cost-based co-expansion planning model, which considered the interactions among wind units, P2G facilities, and GSSs in the expansion planning problem of G\&ES [23]. The results indicate that GSSs have a prominent role in coordinating the ESs by accommodating wind power and balancing temporal energy needs. Findings also reveal that GSSs together with $\mathrm{P} 2 \mathrm{G}$ and wind units can make up a promising combination for alleviating the problems arising from the lack of NG. A similar framework was pursued in [24], which developed an expansion co-planning model for integrated power and gas systems with respect to NG storage and P2G facilities. From the obtained results and discussions provided in this work, it can be inferred that the promotion of NG conversion and storage systems will be an important step toward realization of green economy and $100 \%$ penetration of RES.

Through a more precise co-expansion planning model, the referenced study [25] scrutinized the importance of GSSs in managing short-term uncertainties in developing a long-term expansion plan for both G\&ESs. By adopting a chance-constrained programming approach, the proposed model aims to minimize the investment cost of under-study ES infrastructure, while the desired confidence level for meeting future stochastic electricity and NG demands is reached. Experimental results demonstrate that GSSs are efficient in managing short-term stochasticity of the demand pairs in the long-term co-planning of the G\&ES and in enhancing the reliability of both ESs. In this work, similar to all the above-reviewed works that had developed an integrated planning model, the heat demand was considered in terms of NG demand, resulting in elimination of the impacts that some generating/converting technologies, such as HPs and CHPs, might have on expansion strategies from the model.

\subsection{Paper aim and structure}

As can be seen from the available literature, how the EH approach can improve expansion plans of gas and electricity ESs by considering all the supply chains' rings of the carriers demanded by end users, i.e., heat, gas, and electricity, in comparison with common co-expansion models, has received less attention so far. Hence, to clarify how the EH concept can affect expansion strategies of G\&ES in a more real, suitable, and qualified manner, this paper aims to concentrate on coordinated expansion planning of heat and electricity supply chains under the umbrella of $\mathrm{EH}$ with respect to all effective rings of the chains. In doing so, a multiregional, multistage, and matrixset-based model formulated based on an MIPL-based problem is presented. GPG units, transmission lines, gas pipelines, gas supply systems, gas stations, and gas reservoir systems along with CHP units, GDG units, gas furnaces, and HPs are included in the chains' rings considered in the expansion planning problem. To achieve more realistic and compatible strategies, the most important and effective aspects of planning of the ESs are incorporated into the model that is accessible to a central decision-making authority in a partially deregulated environment. Among these aspects, the contribution of the private sector to investing in RESbased units in terms of Independent Power Producers (IPPs), incentive-based energy policies, budget constraint, and climate characteristics affecting RES-based units' capacity factor is outstanding.

In line with the main objective, this study is organized as follows. Section 2 explores the concept of $\mathrm{EH}$ prior to forming a general model of the typical EH. Section 3 constructs the proposed optimization framework. Section 4 deals with the numerical analysis in which the pursued framework is implemented on the test systems. Section 5 provides the drawn conclusion.

\section{The EH: A step toward sustainable development}

Various studies have presented diverse definitions and models for the $\mathrm{EH}$ concept [6,4-13]. In [6] which is among to first works that has applied EH, an energy hub was defined as a set of interface equipment between energy sources and end-users. Thus, it can be said 
that the $\mathrm{EH}$ is not a new system or infrastructure among existing ESs. It is indeed a novel and general viewpoint on ESs in planning problems while minimum dependency between ESs, especially between G\&ES, has always existed and the possibility of converting different forms of energy into each other has been provided through technological progresses since many years ago.

To form a proper model of infrastructures supplying NG, thermal, and electrical loads, it is required to focus on the supply chain rings of these carriers. Regarding the structure of an NG system, gas wells, GSSs, pipelines, compressors, and demand-side convertors can be treated as the basic rings of the $\mathrm{NG}$ and thermal supply chains. Similarly, power plants, transmission networks, and distribution systems are included in the main rings of the electricity supply chain. Now, a comprehensive expansion planning model based on the $\mathrm{EH}$ concept is reached if not only all basic rings but also the interaction between the carriers as well as the possibility of converting them into each other are considered in modeling energy carrier supply chains. In other words, neglecting the final form of energy demanded as a result of neglecting the possibility of converting energy carriers into each other (in particular in demand side) or eliminating a ring of the supply chain of the demanded carries can disrupt the realization of $\mathrm{EH}$ concept in the planning. For instance, in the case of the thermal supply chain, when demanded heat energy is considered in terms of an equivalent value of NG demand in the planning process, G\&ES cannot be completely modeled in terms of an EH. Such a consideration indeed is the reason why the role of some basic rings of the thermal supply chain, such as CHP and HPs, is eliminated from the model, while these energy converters can provide various options to supply the heat demand.

To formulate the EH concept, suppose that the sets $\mathrm{I}=(\alpha, \beta, \ldots, \psi)$ and $\mathrm{O}=(\alpha, \beta, \ldots, \omega)$ represent final energy forms demanded at the output of $\mathrm{EH}$ and input energy forms, respectively. Regarding the type and number of converters considered in the hub, its performance in changing/converting input energy carriers at the output can be mathematically modeled by a matrix, namely mapping matrix, as in Eq. (1):

$$
\begin{aligned}
& \underbrace{\left[\begin{array}{c}
L_{\alpha} \\
L_{\beta} \\
\vdots \\
L_{\psi}
\end{array}\right]}_{\mathrm{L}}=\underbrace{\left[\begin{array}{cccc}
m_{\alpha, \alpha} & m_{\beta, \alpha} & \cdots & m_{\omega, \alpha} \\
m_{\alpha, \beta} & m_{\beta, \beta} & \cdots & m_{\omega, \beta} \\
\vdots & \vdots & & \vdots \\
m_{\alpha, \psi} & m_{\beta, \psi} & \cdots & m_{\omega, \psi}
\end{array}\right]}_{\mathrm{M}} \times \underbrace{\left[\begin{array}{c}
P_{\alpha} \\
P_{\beta} \\
\vdots \\
P_{\omega}
\end{array}\right]}_{\mathrm{P}} \\
& 0 \leq m_{i, j} \leq 1 \quad \forall i \in \mathrm{I}, \quad j \in \mathrm{O} .
\end{aligned}
$$

Each mapping matrix element, named mapping factor, indeed represents performance efficiency of one of the supply chain rings. In this respect, if $P_{\alpha}$ and $L_{\alpha}$ depict electrical energy carrier at the hub input and the relevant load at output, respectively, and the substation transformer is considered as a converter which supplies a part of demanded electrical energy at the output, $m_{\alpha, \alpha}$ indicates the transformer (substation) efficiency as a converter with the same form of input-output energy. Therefore, for each type of demand at the hub output, all possible load supplying strategies are obtained from summing up the multiplication of input carriers to the relevant converters' efficiencies.

Accordingly, in the case of an $\mathrm{EH}$ with multiple input-output, the mapping factor of each input carrier at the output is equal to multiplication of the efficiencies of all converters (rings) existing in the pathway between the input and output. Moreover, each input carrier can be divided into two or more converters. To determine the converter share of each input carrier, a factor known as dispatch factor, $\sigma$, is employed. Eq. (1) can be consequently rewritten as Eq. (2):

$$
\underbrace{\left[\begin{array}{c}
L_{\alpha} \\
L_{\beta} \\
\vdots \\
L_{\psi}
\end{array}\right]}_{\mathrm{L}}=
$$$$
\underbrace{\left[\begin{array}{cccc}
\sigma_{\alpha \rightarrow \alpha}^{c} \cdot \eta_{\alpha \rightarrow \alpha}^{c} & \sigma_{\beta \rightarrow \alpha}^{c} \cdot \eta_{\beta \rightarrow \alpha}^{c} & \cdots & \sigma_{\omega \rightarrow \alpha}^{c} \cdot \eta_{\omega \rightarrow \alpha}^{c} \\
\sigma_{\alpha \rightarrow \beta}^{c} \cdot \eta_{\alpha \rightarrow \beta}^{c} & \sigma_{\beta \rightarrow \beta}^{c} \cdot \eta_{\beta \rightarrow \beta}^{c} & \cdots & \sigma_{\omega \rightarrow \beta}^{c} \cdot \eta_{\omega \rightarrow \beta}^{c} \\
\vdots & \vdots & & \vdots \\
\sigma_{\alpha \rightarrow \psi}^{c} \cdot \eta_{\alpha \rightarrow \psi}^{c} & \sigma_{\beta \rightarrow \psi}^{c} \cdot \eta_{\beta \rightarrow \psi}^{c} & \cdots & \sigma_{\omega \rightarrow \psi}^{c} \cdot \eta_{\omega \rightarrow \psi}^{c}
\end{array}\right]}_{\mathrm{M}}
$$$$
\times \underbrace{\left[\begin{array}{c}
P_{\alpha} \\
P_{\beta} \\
\vdots \\
P_{\omega}
\end{array}\right]}_{\mathrm{P}} ; c \in \mathbb{C}=\{C H P, F u r, T R, \ldots\}
$$

$$
\begin{aligned}
\sum_{i \in \mathrm{I}, c \in \mathbb{C}} \sigma_{\alpha \rightarrow i}^{c} & =\sum_{i \in \mathrm{I}, c \in \mathbb{C}} \sigma_{\beta \rightarrow i}^{c}=\ldots \\
& =\sum_{i \in \mathrm{I}, c \in \mathbb{C}} \sigma_{\omega \rightarrow i}^{c}=1,
\end{aligned}
$$

where $\sigma_{j \rightarrow i}^{c}$ is the percentage of input carrier $j$ received by a converter of the hub converters set $\mathbb{C}$, converting the input carrier into the output $i$. The sum of dispatch factors of each input carrier should be equal to 1 , formulated in Eq. (3). By limiting input carriers to gas and electricity carriers and output ones to heat and electricity forms, Figure 1 shows a typical gaselectricity hub. Regarding the performance efficiency of each ring shown in the figure, meeting output thermal and electrical loads can be formulated as Eq. (4), 
$\left[\begin{array}{c}L_{e} \\ L_{t h}\end{array}\right]=\mathrm{M}\left[\begin{array}{c}P_{e} \\ P_{g}\end{array}\right] ;$ if $\eta_{g \rightarrow t h}^{G S} \approx 1 ; \mathrm{M}=\left[\begin{array}{cc}\eta_{e \rightarrow e}^{S T} & \left(\begin{array}{c}\sigma_{g \rightarrow e}^{G P G} \cdot \eta_{g \rightarrow e}^{G P G} \cdot \eta_{e \rightarrow e}^{T L} \cdot \eta_{e \rightarrow e}^{S T}+ \\ \sigma_{g \rightarrow e, t h}^{C H P} \cdot \eta_{g \rightarrow e}^{C H P}+\sigma_{g \rightarrow e, t h}^{G P G} \cdot \eta_{g \rightarrow e}^{G P G}\end{array}\right) \eta_{g \rightarrow g}^{C O} \cdot \eta_{g \rightarrow g}^{G P} \\ \eta_{e \rightarrow e}^{S T} \cdot \eta_{e \rightarrow t h}^{H P} & \left(\sigma_{g \rightarrow t h}^{F u r} \cdot \eta_{g \rightarrow t h}^{F u r}+\sigma_{g \rightarrow e, t h}^{C H P} \cdot \eta_{g \rightarrow t h}^{C H P}\right) \eta_{g \rightarrow g}^{C O} \cdot \eta_{g \rightarrow g}^{G P}\end{array}\right]$.

Box I

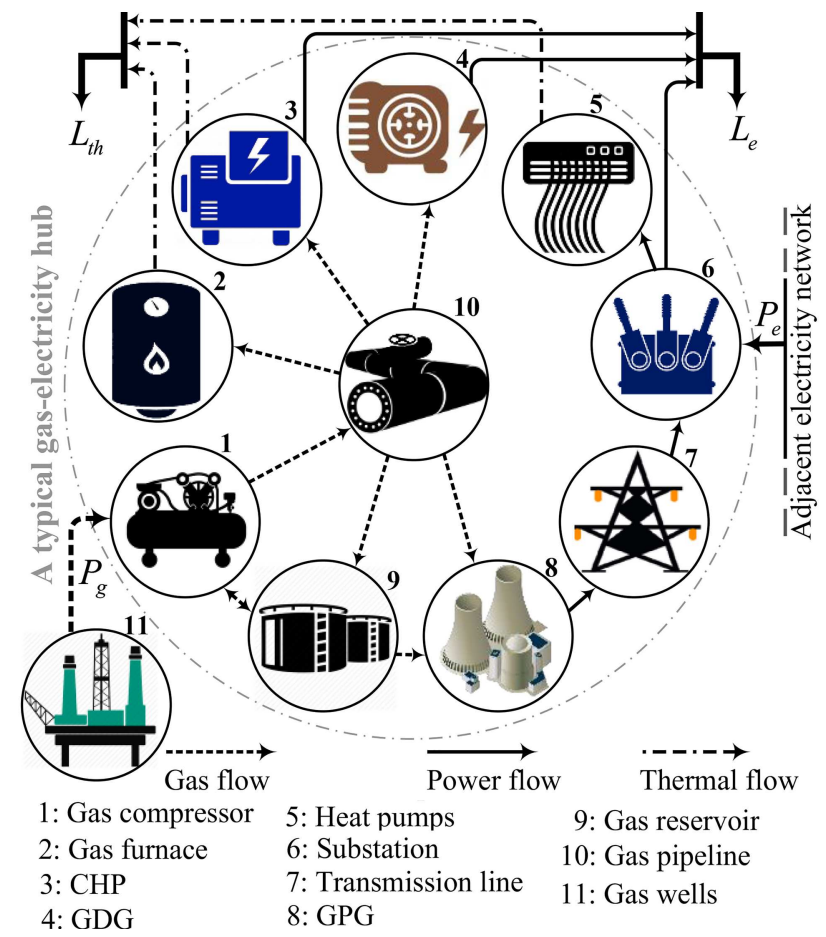

Figure 1. A typical gas-electricity energy hub.

as shown in Box I, in which $\eta_{g \rightarrow g}^{G P}$ and $\eta_{e \rightarrow e}^{T L}$ depict the efficiency of gas pipeline and transmission line, respectively, as the losses of these infrastructures are regarded in terms of their efficiency.

\section{Modeling procedure of G\&ES co-expansion planning under the umbrella of EH}

In this section, a multistage, multiregional, and matrixset-based model is proposed to plan expansion strategies of G\&ES infrastructures in the framework of $\mathrm{EH}$ so that all capital and operation costs are minimized; meanwhile, the required techno-economic constraints are satisfied. The model formulated in terms of a Mixed Integer Linear Programming (MILP) problem is tailored for a semi-deregulated environment to incorporate the role of RES-based IPPs into the model. Gas supply systems (set of gas wells and refineries), GSSs, gas pipelines, GPGs, and transmission lines are the main infrastructures regarded in generation and transmission sectors. GDG units, gas furnaces, and HPs along with RES-based units (in term of IPPs) are encompassed technology options considered on the demand side to convert input energy carriers into the desirable forms. Different parts of the proposed model are presented in the following.

\subsection{Objective function}

The Objective Function (OF) is comprised of the Net Present Value (NPV) of all G\&ES costs to be minimized. As can be seen in Eq. (5), the OF has 24 cost terms in $(\mathrm{M} \$)$ defined by Eqs. (6) and (7) corresponding to the heat and electricity supply chains, respectively. For instance, in the case of the thermal supply chain, the first term, i.e., $\Gamma_{1}^{H}$, depicts the investment cost of the $n$th gas supply system from type $i$ in region $s$ and the $t$ th stage of the planning horizon.

$$
\begin{aligned}
& \text { Min }: O F=\sum_{t=1}^{T} \sum_{s=1}^{S}(1+r)^{1-t} \\
& {[\underbrace{\left(\Gamma_{1}^{H}+\Gamma_{2}^{H}+\ldots+\Gamma_{13}^{H}\right)}_{\Gamma^{H}}+\underbrace{\left(\Gamma_{1}^{E}+\Gamma_{2}^{E}+\ldots+\Gamma_{11}^{E}\right)}_{\Gamma^{E}}] \cdot } \\
& \Gamma^{H}=\sum_{i \in I^{S P}(s)} \sum_{n=1}^{n^{S P}(i)}\left(\begin{array}{l}
C_{i n v}^{S P}(i, s, n, t) \cdot \\
x^{S P}(i, s, n, t)
\end{array}\right) \\
&+\sum_{k=1, k \neq s}^{S} \sum_{i \in I^{G P}} \sum_{n=1}^{n^{G P}(i)}\left(\begin{array}{l}
0.5 C_{i n v}^{G P}(i, s, k, n, t) \cdot \\
x^{G P}(i, s, k, n, t)
\end{array}\right) \\
&+\sum_{i \in I^{G S}(s)} \sum_{n=1}^{n^{G S}(i)}\left(\begin{array}{l}
C_{i n v}^{G S}(i, s, n, t) \cdot \\
x^{G S}(i, s, n, t)
\end{array}\right) \\
&+\sum_{i \in I^{F U}(s)} \sum_{n=1}^{n^{F U}(i)}\left(\begin{array}{l}
C_{i n v}^{F U}(i, s, n, t) \cdot \\
x^{F U}(i, s, n, t)
\end{array}\right) \\
&+\sum_{i \in I^{C O}(s)} \sum_{n=1}^{n_{n}^{C O}(i)}\left(\begin{array}{l}
C_{i n v}^{C O}(i, s, n, t) \cdot \\
x^{C O}(i, s, n, t)
\end{array}\right) \\
&+\sum_{i \in I^{H P}(s)} \sum_{n=1}^{n^{H P}(i)}\left(\begin{array}{l}
C_{i n v}^{H P}(i, s, n, t) \cdot \\
x^{H P}(i, s, n, t)
\end{array}\right)
\end{aligned}
$$




$$
\begin{aligned}
& +\sum_{i \in I_{N \& E}^{G S}(s, t)} \sum_{n=1}^{n_{I O}^{G S}(i, s, t)} \sum_{b=1}^{B} \\
& \left(\begin{array}{c}
\mu(i, s, n, t) \cdot v^{i n j}(i, s, n, b, t) \\
+\lambda(i, s, n, t) \cdot v^{w i t h}(i, s, n, b, t)
\end{array}\right) \cdot \Phi^{\overline{\mathrm{G}}}(s, b, t) \\
& +\sum_{i \in I_{N \& E}^{G S}(s, t)} \sum_{n=1}^{n_{I O}^{G S}(i, s, t)} C_{O \& M}^{G S}(i, s, n, t) \cdot \bar{v}(i) \\
& +\sum_{i \in I_{N \& E}^{S P}(s, t)} \sum_{n=1}^{n_{I O}^{S P}(i, s, t)}\left(\begin{array}{l}
C_{O \& M}^{S P}(i, s, n, t) \cdot \\
\bar{g}^{S P}(i, s, n, t)
\end{array}\right) \\
& +\sum_{i \in I_{N \& E}^{S P}(s, t)} \sum_{n=1}^{\substack{S P \\
I O}} \sum_{b=1}^{B} C_{o p r}^{S P}(i, s, s, n, t) \\
& \cdot g^{S P}(i, s, n, b, t) \cdot \Phi^{\overline{\mathrm{G}}}(s, b, t) \\
& +\sum_{i \in I_{N \& E}^{C O}(s, t)} \sum_{n=1}^{n_{I O}^{C O}(i, s, t)} \sum_{b=1}^{B} C_{o p r}^{C O}(i, s, n, t) \\
& \cdot G c(i, s, n, b, t) \cdot \Phi^{\overline{\mathrm{G}}}(s, b, t) \\
& +\sum_{i \in I_{N \in E}^{F U}(s, t)} \sum_{n=1}^{n_{I O}^{F U}(i, s, t)} \sum_{b=1}^{B} C_{o p r}^{F U}(i, s, n, t) \\
& \cdot h^{F U}(i, s, n, b, t) \cdot \Phi^{\mathrm{H}}(s, b, t) \\
& +\sum_{i \in I_{N \& E}^{H P}(s, t)} \sum_{n=1}^{n_{I O}^{H P}(i, s, t)} \sum_{b=1}^{B} C_{o p r}^{H P}(i, s, n, t) \\
& \cdot h^{H P}(i, s, n, b, t) \cdot \Phi^{\mathrm{H}}(s, b, t), \\
& \Gamma^{E}=\sum_{i \in I^{G P G}(s)} \sum_{n=1}^{n^{G P G}(i)}\left(\begin{array}{l}
C_{i n v}^{G P G}(i, s, n, t) \cdot \\
x^{G P G}(i, s, n, t)
\end{array}\right) \\
& +\sum_{i \in I^{G P G}(s)} \sum_{n=1}^{n^{C H P}(i)}\left(\begin{array}{l}
C_{i n v}^{C H P}(i, s, n, t) \cdot \\
x^{C H P}(i, s, n, t)
\end{array}\right) \\
& +\sum_{i \in I^{G D G}(s)} \sum_{n=1}^{n^{G D G}(i)}\left(\begin{array}{c}
C_{i n v}^{G D G}(i, s, n, t) \cdot \\
x^{G D G}(i, s, n, t)
\end{array}\right) \\
& +\sum_{k=1, k \neq s}^{S} \sum_{n=1}^{n_{s-k}^{T L}}\left(\begin{array}{l}
0.5 C_{i n v}^{T L}(s, k, n, t) \cdot \\
x^{T L}(s, k, n, t)
\end{array}\right)
\end{aligned}
$$

$$
\begin{aligned}
& +\sum_{i \in I_{N \& E}^{G P G}(s, t)} \sum_{n=1}^{n_{I O}^{G P G}(i, s, t)}\left(\begin{array}{l}
C_{O \& M}^{G P G}(i, s, n, t) \cdot \\
\bar{p}^{G P G}(i, s, n, t)
\end{array}\right) \\
& +\sum_{i \in I_{N \& E}^{C H P}(s, t)} \sum_{n=1}^{n_{I O}^{C H P}(i, s, t)}\left(\begin{array}{l}
C_{O \& M}^{C H P}(i, s, n, t) \cdot \\
\bar{p}^{C H P}(i, s, n, t)
\end{array}\right) \\
& +\sum_{i \in I^{R E}(s)} \sum_{n=1}^{n^{R E}(i)} \\
& \left(\begin{array}{c}
C_{i n c}^{R E}(i, s, n, t) \cdot \bar{p}^{R E}(i, s, n, t) \cdot \\
\delta(i, s) \cdot x^{R E}(i, s, n, t)
\end{array}\right) \\
& +\sum_{b=1}^{B}\left(\begin{array}{c}
\operatorname{VOLL}(s, b, t) \cdot \\
\operatorname{EENS}(s, b, t)
\end{array}\right) \cdot \Phi^{\mathrm{E}}(s, b, t) \\
& +\sum_{i \in I_{N \& E}^{G P G}(s, t)} \sum_{n=1}^{n_{I O}^{G P G}(i, s, t)} \sum_{b=1}^{B} C_{o p r}^{G P G}(i, s, n, t) \\
& \cdot p^{G P G}(i, s, n, b, t) \cdot \Phi^{\mathrm{E}}(s, b, t) \\
& +\sum_{i \in I_{N \& E}^{C H P}(s, t)} \sum_{n=1}^{n_{I O}^{C H P}(i, s, t)} \sum_{b=1}^{B} C_{o p r}^{C H P}(i, s, n, t) \\
& \cdot p^{C H P}(i, s, n, b, t) \cdot \Phi^{\mathrm{E}}(s, b, t) \\
& +\sum_{i \in I_{N \& E}^{G D G}(s, t)} \sum_{n=1}^{n_{I O}^{G D G}(i, s, t)} \sum_{b=1}^{B} C_{o p r}^{G D G}(i, s, n, t) \\
& \cdot p^{G D G}(i, s, n, b, t) \cdot \Phi^{\mathrm{E}}(s, b, t) .
\end{aligned}
$$

Investment costs of gas pipelines installed between regions $s$ and $k, n$th GSS of type $i$ in the relevant region and planning stage, gas furnaces, gas compressors, and HPs are represented by the 2nd-6th terms in Eq. (6), respectively. Variable operation cost of GSSs, comprising injection and withdrawal costs, the relevant fixed Operation and Maintenance (O\&M) costs, the O\&M cost of gas supply systems, their variable operation costs, and the variable operation costs of gas compressors are given by the last five terms, respectively. Similar to Eq. (6), Eq. (7) represents the costs associated with expansion of the NG-based supply chain of electricity carrier. The first four terms show the capital costs of GPG, CHP, and GDG units plus capital cost of TL, respectively. The fifth and sixth terms are indeed the O\&M costs of GPG and CHP units, respectively. The costs derived from the considered incentive measure, i.e., Feed-In-Tariff 
(FIT), to encourage IPPs to invest in RES-based units and from Expected Energy Not Supplied (EENS) during each planning stage are modeled by the seventh and eighth terms of Eq. (7), respectively. Ultimately, operation costs of all generating units are considered by the last three terms of the aforementioned equation.

\subsection{Demand modeling}

At the heart of ESs planning problems is Load Duration Curve (LDC). The LDC is obtained by sorting the loads from the largest to smallest value, resulting in the loss of the chronological information. It indeed illustrates the frequency that a particular level of demand appears in a year. For large-scale and long-term problems, the load duration curve can be simplified using linear approximation. Hence, the yearly demand is modeled by three load blocks using the base, intermediate (mid), and peak hours of a typical LDC at each node. Moreover, similar to electricity demand, demand behaviors for the other carriers under study in each region are modeled as the discretized LDC, as shown in Figure 2 for NG load. In this figure, the gas supply systems are allocated to meet the base, medium, and peakload demands during the planning horizon. $\Phi^{G}(s, B)$, $\Phi^{G}(s, M)$, and $\Phi^{G}(s, P)$ denote the time periods of base, medium, and peak load demands.

\subsection{NG system constraints}

Regarding the first six terms in Eq. (6) representing the cost terms of gas and heat supply chains, the operation and investment costs of each infrastructure are taken into account when the state of relevant binary variable changes to 1 . Moreover, the state of each binary variable is not changeable until the end of the planning horizon unless the infrastructure is decommissioned because of the lifetime constraint, if considered. The capital cost of each infrastructure is assumed to be added to the OF at the beginning of the relevant operation period. Furthermore, to compute operation costs in each stage, the number of in-operation infrastructures should be updated. The binary variables are defined by Eqs. (8) and (9):

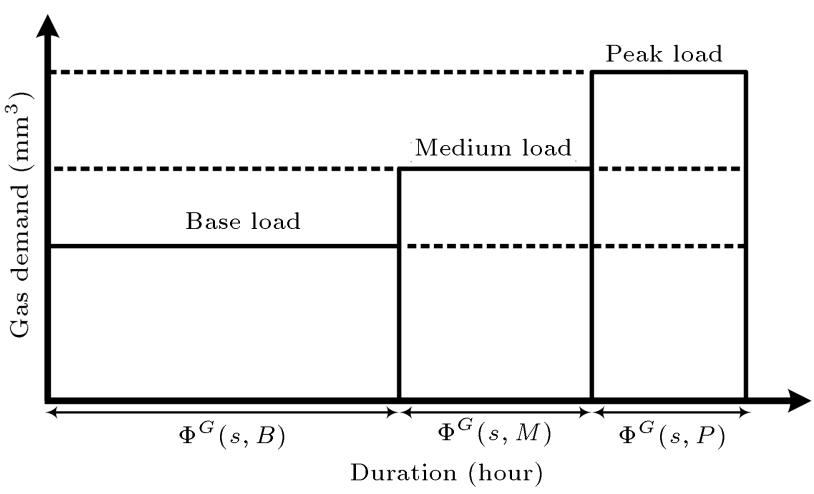

Figure 2. The discretized NG load duration curve.

$$
\begin{gathered}
x^{i n f r a}(i, s, n, t) \in\{0,1\} ; t=1, . ., T ; s=1, . ., S ; \\
n=1, . ., n_{I O}^{i n f r a}(i, s, t) ; \forall i \in I_{N \& E}^{\text {infra }}(s, t) ; \\
\forall i n f r a \in\{S P, G S, C O, F U, H P\}, \\
x^{G P}(i, s, k, n, t) \in\{0,1\} ; t=1, . ., T ; s=1, . ., S ; \\
k=1, . ., S, k \neq s ; \\
n=1, . ., n_{I O}^{G P}(i, s, k, t) ; \forall i \in I_{N \& E}^{G P}(s, k, t) .
\end{gathered}
$$

Moreover, for a set of the considered NG system infrastructures, constraints on the state of binary variables and updating the number of infrastructures are formulated by Eqs. (10)-(11) and (12)-(13), respectively:

$$
\begin{aligned}
& x^{i n f r a}(i, s, n, t) \leq x^{i n f r a}(i, s, n, t+1) ; t=1, . ., T \\
& s=1, . ., S \\
& n=1, . ., n_{I O}^{i n f r a}(i, s, t) ; \forall i \in I_{N \& E}^{i n f r a}(s, t) ; \\
& \forall i n f r a \in\{S P, G S, C O, F U, H P\} \text {, } \\
& x^{G P}(i, s, k, n, t) \leq x^{G P}(i, s, k, n, t+1) ; t=1, . ., T \\
& s=1, . ., S ; k=1, . ., S \\
& n=1, . ., n_{I O}^{G P}(i, s, k, t) ; \forall i \in I_{N \& E}^{G P}(s, k, t), \\
& n_{I O}^{i n f r a}(i, s, t+1)=n_{I O}^{i n f r a}(i, s, t)+\sum_{n} x^{i n f r a}(i, s, n, t) ; \\
& t=1, \ldots, T ; \quad s=1, \ldots, S \\
& \forall i \in I_{N \& E}^{i n f r a}(s, t) \\
& \forall \text { infra } \in\{S P, G S, C O, F U, H P\} \text {, } \\
& n_{I O}^{G P}(i, s, k, t+1)=n_{I O}^{G P}(i, s, k, t) \\
& +\sum_{n} x^{G P}(i, s, k, n, t) \\
& t=1, \ldots, T ; s=1, \ldots, S ; k=1, \ldots . S ; k \neq s ; \\
& \forall i \in I_{N \& E}^{G P}(s, k, t) .
\end{aligned}
$$

Apart from the number of infrastructures, the sets encompassing technology types of each infrastructure should be also upgraded as formulated by Eqs. (14) and (15): 


$$
\begin{aligned}
& I_{N \& E}^{\text {infra }}(s, t+1)=I_{N \& E}^{\text {infra }}(s, t) \cup\{i\}, \forall i \in I^{\text {infra }}(s), \\
& \quad \text { if } x^{i n f r a}(i, s, n, t)=1 ; n=1, . ., n^{i n f r a}(i) ; \\
& \quad t=1, . ., T ; s=1, . ., S ; \\
& \forall i n f r a \quad \in\{S P, G S, C O, F U, H P\}, \\
& I_{N \& E}^{G P}(s, k, t+1)=I_{N \& E}^{G P}(s, k, t) \cup\{i\}, \forall i \in I^{G P}(s, k), \\
& \quad \text { if } x^{G P}(i, s, k, n, t)=1 ; n=1, . ., n^{G P}(i) ; \\
& \quad t=1, . . T ; s=1, . ., S ; k=1, . ., S ; k \neq s .
\end{aligned}
$$

The restrictions on the installation time of each infrastructure of the NG system are incorporated into the model in the following:

$$
\begin{gathered}
\sum_{n=1}^{n_{I O}^{i n f r a}(i, s, t)} x^{i n f r a}(i, s, n, t) \leq \bar{N}^{i n f r a}(i, s, t) ; \\
t=1, . ., T ; s=1, . ., S ; i \in I^{i n f r a}(s) ; \\
\forall i n f r a \in\{S P, G S, C O, F U, H P\}, \\
\sum_{n=1}^{n_{I O}^{G P}(i, s, k, t)} x^{G P}(i, s, k, n, t) \leq \bar{N}^{G P}(i, s, k, t) ; \\
t=1, . ., T ; s=1, . ., S ; \quad k=1, . ., S, \\
k \neq s ; i \in I^{G P} .
\end{gathered}
$$

GSSs, in particular Under Ground Storage (UGS) ones, play a prominent role in load meeting during the cold periods of years and in postponing investment costs of NG systems expansion capacity. The operation of GSSs is modeled with respect to their storage volume limit as well as the limitations of NG injection and withdrawal values. The typical representation of the above-ground NG reservoir is given in Figure 3. As can be seen from this figure, a GSS characterized by a fixed volume in specific pressure has minimum $(\underline{v}(i))$

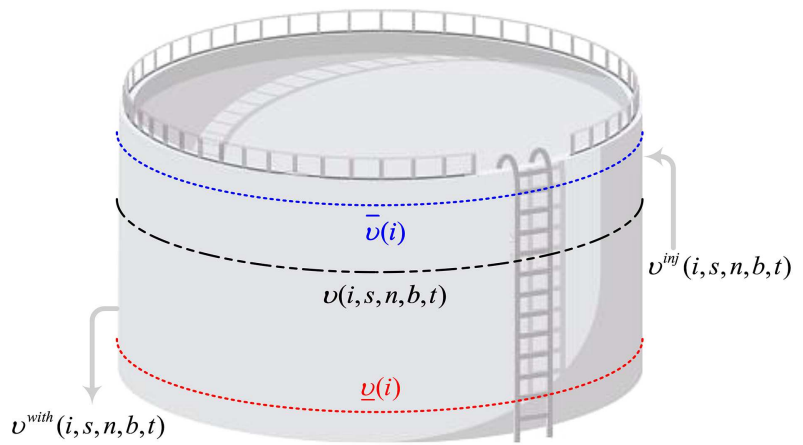

Figure 3. Schematic of a gas storage system. and maximum $(\bar{v}(i))$ capacity storages as well as limit injection $\left(v^{i n j}\right)$ and withdrawal $\left(v^{\text {with }}\right)$ volumes per hour. The volume of each GSS in load block $b$ can be expressed as Eq. (18) [14]. The constraints on storage capacity and injection/withdrawal processes are also considered by Eqs. (19)-(21):

$$
\begin{aligned}
& v(i, s, n, b, t)=v(i, s, n, b-1, t)+\left(v^{i n j}(i, s, n, b, t)\right. \\
& \left.-v^{\text {with }}(i, s, n, b, t)\right) \cdot \Phi^{\overline{\mathrm{G}}}(s, b, t) ; s=1, . ., S \\
& b=1, . ., B ; t=1, . ., T ; n=1, . ., n_{I O}^{G S}(i, s, t) ; \\
& \forall i \in I_{N \& E}^{G S}(s, t) \\
& v(i, s, n, b, t) \leq \bar{v}(i) \& \quad v(i, s, n, b, t) \geq \underline{v}(i) \\
& s=1, . ., S ; b=1, \ldots, B ; t=1, . ., T \\
& n=1, . ., n_{I O}^{G S}(i, s, t) ; \forall i \in I_{N \& E}^{G S}(s, t) \\
& v^{i n j}(i, s, n, b, t) \leq \bar{v}^{i n j}(i) ; \quad \& \quad v^{i n j}(i, s, n, b, t) \\
& \geq \underline{v}^{i n j}(i) ; s=1, . ., S ; b=1, \ldots, B ; t=1, . ., T ; \\
& n=1, . ., n_{I O}^{G S}(i, s, t) ; \forall i \in I_{N \& E}^{G S}(s, t), \\
& v^{\text {with }}(i, s, n, b, t) \leq \bar{v}^{\text {with }}(i) ; \& v^{\text {with }}(i, s, n, b, t) \\
& \geq \underline{v}^{\text {with }}(i) ; s=1, . ., S ; b=1, \ldots, B ; t=1, . ., T ; \\
& n=1, . ., n_{I O}^{G S}(i, s, t) ; \forall i \in I_{N \& E}^{G S}(s, t) .
\end{aligned}
$$

Complete modeling of the performance of GSSs is contingent upon the consideration of GSSs' storing capability during base and medium load periods at each stage. In doing so, if $v^{G S}(s, b, t)$ depicts the volume of stored NG in region $s$ during the load block $b$, the difference between NG supply capacity plus received volume and the total of transmitted plus consumed capacities can be stored in the GSS as follows:

$$
\begin{aligned}
& v^{G S}(s, b, t)=v^{G S}(s, b-1, t) \\
& +\left(\sum _ { i \in I _ { N \& E } ^ { G S } ( s , t ) } \sum _ { n = 1 } ^ { n _ { I O } ^ { G S } ( i , s , t ) } \left(v^{i n j}(i, s, n, b, t)\right.\right. \\
& \left.\left.-v^{w i t h}(i, s, n, b, t)\right)\right) \cdot \Phi^{\overline{\mathrm{G}}}(s, b, t) ; \\
& s=1, . ., S ; b=1, . ., B ; t=1, . ., T \\
& v^{G S}(s, b, t) \geq \tau(b) \cdot \bar{v}^{G S}(s, t) ; \text { if }
\end{aligned}
$$




$$
\begin{gathered}
\sum_{i \in I_{N \& E}^{S P}(s, t)} \sum_{n=1}^{\substack{S P \\
I O}} \bar{g}^{S P}(i, n)+P_{g}^{i n j}(s, b, t) \\
\geq \Omega^{\overline{\mathrm{G}}}(s, b, t) ; s=1, \ldots, S ; b=1, \ldots, b^{\text {peak }} ; \\
t=1, \ldots, T, \\
\frac{v^{G S}(s, b, t)}{\bar{v}^{G S}(s, t)} \leq \tau(b) \leq 1 ; s=1, \ldots, S ; b=1, \ldots, b^{\text {peak }} ; \\
t=1, \ldots, T .
\end{gathered}
$$

where $P_{g}^{i n j}(s, b, t)$ represents total hourly NG volume injected into region $s ; \tau(b)$ is the charge coefficient with a positive slope which is the reason why the GSS is charged during the base- and medium-load periods if the capacity restriction of the relevant gas supply system and the pipeline is allowed. As a matter of fact, increase in the aforementioned coefficient results in the variation of the injection variable, i.e., $v^{i n j}(i, s, n, b, t)$, in the defined range with respect to the load fluctuations (demands and stations consumption values) of all other regions that are jointly supplied by the relevant gas supply system along with the region $s$. Accordingly, from the beginning of the base-load period, i.e., $b=1$, to the beginning of peak-load period, i.e., $b=b^{\text {peak }}$ in each stage, $\tau(b)$ may vary in direct proportion to gas demands and increase the volume of stored gas to the highest possible level.

The capacity constraints of gas supply systems, pipelines, and gas compressors are taken into account by Eqs. (25)-(27):

$$
\begin{aligned}
& g^{S P}(i, s, n, b, t) \leq \bar{g}^{S P}(i) ; \quad g^{S P}(i, s, n, b, t) \\
& \geq \underline{g}^{S P}(i) ; s=1, . ., S ; b=1, . ., B ; t=1, . ., T ; \\
& n=1, . ., n_{I O}^{S P}(i, s, t) ; \quad \forall i \in I_{N \& E}^{S P}(s, t), \\
& \left|f_{g}(i, s, k, n, b, t)\right| \leq \overline{f_{g}}(i) ; s=1, . ., S ; k=1, . ., S \text {; } \\
& k \neq s ; b=1, . ., B ; t=1, . ., T \\
& n=1, . ., n_{I O}^{G P}(i, s, k, t) ; i \in I^{G P}, \\
& \underbrace{\sum_{i \in I_{N \& E}^{G P}(s, k, t)} \sum_{n=1}^{n_{I O}^{G P}(i, s, k, t)} f_{g}^{+}(i, s, k, n, b, t)}_{\widetilde{F_{g}}(s, k, b, t)} \\
& \leq \sum_{i \in I_{N \& E}^{C O}(s, t)} \sum_{n=1}^{n_{I O}^{C O}(i, s, t)} \bar{g}^{C O}(i, s, k, n, t)
\end{aligned}
$$

$$
\begin{aligned}
& s=1, . ., S ; k=1, . ., S ; k \neq s ; b=1, . ., B ; \\
& t=1, . ., T
\end{aligned}
$$

where $\widetilde{F_{g}}(s, k, b, t)$ is the total transmitted NG volume from region $s$ to $k$ and $f_{g}(i, s, k, n, b, t)$ is the pipeline gas flow variable, which can take either positive or negative values with regard to the flow direction [14].

In gas stations, the required electricity for gas compressors is usually supplied by located DG units consuming around $3 \%-5 \%$ of the transmitted gas depending upon the compressor horse power, which in turn is a function of flow rate and the ratio of inputoutput gas pressures, as shown in empirical Eq. (28) [17] as follows:

$$
\begin{gathered}
P c(i, s, k, n, b, t)=\frac{\widetilde{F_{g}}(s, k, b, t) \cdot \beta(i, s, n, t) \cdot \varphi}{\eta^{C O}(i) \cdot(\varphi-1)} \\
{\left[\left(\frac{\rho^{\text {out }}(i, s, n, b, t)}{\rho^{i n}(i, s, n, b, t)}\right)^{\frac{(\varphi-1)}{\varphi}}-1\right] ;} \\
s=1, . ., S ; t=1, . ., T ; b=1, . ., B ; \\
n=1, . ., n_{I O}^{C O}(i, s, t) ; \forall i \in I_{N \& E}^{C O}(s, t), \\
n_{I O}^{G P}(i, s, k, t) \\
\sum_{n=1}^{G P}(i, s, k, n, t) \leq \bar{N}^{G P}(i, s, k, t) ; \\
t=1, . ., T ; s=1, . ., S ; k=1, . ., S, \\
k \neq s ; i \in I^{G P},
\end{gathered}
$$

where $\beta(i, s, k, n, t)$ is the participation factor of compressors in boosting the pressure of gas flow transmitted from area $s$ to $k$. Consequently, the NG volume consumed by each station is:

$$
\begin{aligned}
& G c(i, s, k, n, b, t)=\frac{3.412 \times P c(i, s, k, n, b, t)}{\eta^{D G}(i) \cdot \zeta(s)} \\
& s=1, . ., S ; k=1, . ., S ; k \neq s ; t=1, . ., T \\
& b=1, . ., B ; n=1, . ., n_{I O}^{C O}(i, s, t) \\
& \forall i \in I_{N \& E}^{C O}(s, t)
\end{aligned}
$$

where $\eta^{D G}(i)$ is the efficiency of GDG located at the station. Note that considering the physical characteristics of given pipelines, the parameters $\rho^{\text {in }}$, $\rho^{\text {out }}$, and $\varphi$ can be estimated. Here, they are incorporated into the model as constants to keep the linearity $[17,26]$.

\subsection{Electricity system constraints}

Based on the enumerated factors in the importance of $\mathrm{NG}$, enacted policies on this carrier, and the pursued 
linchpin, only NG-based generation technologies are considered as the generation sector of the electricity system. In doing so, among all conventional generation technology options on the generation side and all nonrenewable DG units, GPG together with CHP and GDG units are only considered in terms of Combined and Open-Cycle Gas Turbine (CCGT and OCGT) units to meet the projected demand. Investing in RES in terms of wind and solar units is another load meeting option incorporated into the model as an opportunity for IPPs supported by FIT system. In the following, the constraints derived from investing and operating procedures of these generation options are modeled.

In case of the electricity sector infrastructure, the definition of binary variables along with the relevant constraints as well as updating infrastructures and upgrading the datasets related to the types of technologies in operation correspond to those in Eqs. (8)(15). Accordingly, transmission lines and other electricity system infrastructures (in fra), defined as the set of $\{G P G, C H P, G D G, R E\}$, correspond to gas pipelines and the set of $\mathrm{NG}$ infrastructures, i.e., $\{S P, G S, C O, F U, H P\}$, respectively. The bounds on the duration time of electricity infrastructure installation are also modeled in a similar fashion to the relevant constraints of the NG sector; hence, this issue has not been considered here. The capacity bounds of infrastructures are given as follows:

$$
\begin{gathered}
p^{G P G}(i, s, n, b, t) \leq \bar{p}^{G P G}(i) ; \& p^{G P G}(i, s, n, b, t) \\
\geq \underline{p}^{G P G}(i) ; \\
s=1, . ., S ; b=1, . ., B ; t=1, . ., T \\
n=1, . ., n_{I O}^{G P G}(i, s, t) ; \forall i \in I_{N \& E}^{G P G}(s, t), \\
p^{C H P}(i, s, n, b, t) \leq \bar{p}^{C H P}(i) ; \& p^{C H P}(i, s, n, b, t) \\
\geq \underline{p}^{C H P}(i) ; \\
s=1, . ., S ; b=1, . ., B ; t=1, . ., T ; \\
\quad n=1, . ., n_{I O}^{C H P}(i, s, t) ; \forall i \in I_{N \& E}^{C H P}(s, t), \\
p^{G D G}(i, s, n, b, t) \leq \bar{p}^{G D G}(i) ; \& p^{G D G}(i, s, n, b, t) \\
\geq \underline{p}^{G D G}(i) ; \\
s=1, . ., S ; b=1, . ., B ; t=1, . ., T ; \\
n=1, . ., n_{I O}^{G D G}(i, s, t) ; \forall i \in I_{N \& E}^{G D G}(s, t) \\
\left|f_{e}(s, k, n, b, t)\right| \cdot x^{T L}(s, k, n, t) \leq \overline{f_{e}} ; s=1, . ., S \\
\quad k
\end{gathered}
$$

$$
\begin{aligned}
& k=1, . ., S ; k \neq s ; b=1, . ., B ; t=1, . ., T ; \\
& n=1, . ., n_{s-k}^{T L} .
\end{aligned}
$$

Considering the basic distinction between power industries of different countries, restructuring has led to the transformation of planning models. In a semideregulated structure, the IPPs sell their generation to the utility acting as a government-dependent purchasing agent. Here, to realistically incorporate the role of RES generators into the model, they are considered as the IPPs supported by the FIT mechanism that is implemented in some developing countries like Iran. It is noteworthy that among various FIT models, the fixed premium model is considered here. In this type of FIT system, a technology-specific environmental premium (bonus) is paid above the normal electricity price to the renewable energy generators mandated by a regulator and guaranteed for a fixed period of time [27]. From the IPP viewpoint, investment in RES-based units is contingent upon the profitability of the project which, in turn, depends on the associated capital costs, the estimated capacity factor taking into consideration the geographical features of the region, and the amount of FIT premiums. To model the guarantee of the investment profitability of IPPs, we have:

$$
\begin{gathered}
C_{i n c}^{R E}(i, s, n, t) \cdot \bar{p}^{R E}(i, s, n, t) \cdot \delta(i, s) \geq(1+\% \alpha) \\
\cdot\left[\frac{r \cdot C_{i n v}^{R E}(i, s, n, t)}{1-(1+r)^{T^{C o n t}}}+C_{o p r}^{R E}(i, s, n, t)\right] \\
s=1, . ., S ; b=1, . ., B ; t=1, . ., T ; \\
n=1, \ldots, n_{I O}^{R E}(i, s, t) ; \forall i \in I_{N \& E}^{R E}(s, t), \\
\frac{C_{i n c}^{R E}(i, s, t) \leq C_{i n c}^{R E}(i, s, n, t) \leq \overline{C_{i n c}^{R E}}(i, s, t) ;}{s=1, . ., S ; t=1, . ., T ; n=1, \ldots, n_{I O}^{R E}(i, s, t)} \\
\forall i \in I_{N \& E}^{R E}(s, t) .
\end{gathered}
$$

Accordingly, IPPs' income from FIT premiums, i.e., the left side of Eq. (35), at each planning stage should be greater than the total costs imposed on IPPs. This is done by $\alpha$ which indeed is a constant between $r$ and 1 . The RES capital costs are computed in the equivalent annualized form, while the RES projects are mostly funded through governmental banks. Furthermore, to provide a trade-off between IPPs' profit and paid subsidies by the planning authority threatened by overfunding risk, the incentives are considered flexibly as in Eq. (36). The average amount of delivered power by IPPs in region $s$, planning stage $t$, and load block $b$ can be computed as follows: 


$$
\begin{aligned}
& p^{R E}(s, b, t)= \\
& \left(\sum_{i \in I_{N \& E}^{R E}(s, t)} \sum_{n=1}^{n_{I O}^{R E}(i, s, t)} \bar{p}^{R E}(i, s, n, t) \cdot \delta(i, s) / 8760\right) \\
& s=1, . ., S ; b=1, . ., B ; t=1, . ., T .
\end{aligned}
$$

Budget constraint is among the other main factors affecting expansion plans [28]. Here, to plan expansion strategies as realistically as possible, the constraint on budget is given as follows:

$$
\begin{aligned}
& \operatorname{Inv}(t) \leq B(t) ; \quad t=1, \ldots, T, \\
& B(t)=(1+r) B(t-1)-\operatorname{Inv}(t) \\
& B(0)=B^{t o t} ; t=1, . ., T, \\
& \operatorname{Inv}(t)=\sum_{j=1}^{6} \Gamma_{j}^{H}+\sum_{q=1}^{4} \Gamma_{q}^{E} ; \quad t=1, \ldots, T .
\end{aligned}
$$

Regarding the eighth term of Eq. (7), it is required that the total amount of EENS at each stage computed by Eq. (41) be limited to a certain value as in Eq. (43). Note that since the values of lost loads in different regions may differ from one another, different EENS caps can be allocated to them in Eq. (42):

$$
\begin{aligned}
& \operatorname{EENS}(s, b, t)=C R(s, b, t) \cdot \Omega^{\mathrm{E}}(s, b, t) \\
& s=1, . ., S ; b=1, . ., B ; t=1, . ., T \\
& \sum_{b=1}^{B} \operatorname{EENS}(s, b, t) \leq \overline{\operatorname{EENS}}(s, t) \\
& \quad s=1, . ., S ; t=1, . ., T \\
& \sum_{s=1}^{S} \sum_{b=1}^{B} E E N S(s, b, t) \leq \overline{\overline{E E N S}}(t) ; t=1, . ., T .
\end{aligned}
$$

\subsection{EH constraints}

Regardless of energy distribution systems, if each region of the system under study is considered in terms of a micro hub (node), the injected amount of each input carrier into each node, i.e., $P_{\alpha}^{i n j}(s, b, t)$, can be computed by Eq. (44) as follows [8]:

$$
\begin{aligned}
& P_{\alpha}^{i n j}(s, b, t)=\mathrm{N}_{\alpha}(s, t) * \mathrm{~F}_{\alpha}(s, b, t) \\
& \quad s=1, . ., S ; b=1, . ., B ; t=1, . ., T
\end{aligned}
$$

where $N_{\alpha}(s, t)$ is the connectivity vector of carrier $\alpha$ for region $s$ and $F_{\alpha}(s, b, t)$ is the corresponding flow vector including line/pipeline flows. The elements of $N_{\alpha}(s, t)$ are, in fact, the binary variables of transport/transmission infrastructures of carrier $\alpha$. To better appreciate the content of Eq. (44), consider NG carrier; if the set of gas pipelines connected to region $s$ until stage $t$, i.e., $I_{N \& E}^{G P}(s, k, t)$, is comprised of $q_{1}$ members, the expanded form of Eq. (44), i.e., Eq. (45) as shown in Box II, presents the volume of NG hourly injected into node $s$ in load block $b$. Accordingly, the sum of injected and generated/produced amounts of gas and electricity carriers of each node, as a micro hub, achieves total available energy to meet the projected loads as follows:

$$
\begin{gathered}
P_{e}(s, b, t)=\mathrm{N}_{e}(s, t) * \mathrm{~F}_{e}(s, b, t)+\mathrm{E}(s, t) * \mathrm{p}_{e}(s, b, t) \\
s=1, . ., S ; b=1, . ., B ; t=1, . ., T \\
P_{g}(s, b, t)=\mathrm{N}_{g}(s, t) * \mathrm{~F}_{g}(s, b, t)+\mathrm{G}(s, t) * \mathrm{p}_{g}(s, b, t) \\
s=1, \ldots, S ; b=1, \ldots, B ; t=1, \ldots, T
\end{gathered}
$$

where $E$ and $G$ vectors are indeed the number of electricity generation units and gas supply systems in operation, respectively; $p_{e}$ and $p_{g}$ are corresponding dispatch vectors at node $s$ at stage $t$ during load block $b$. All vectors $E, G, N_{e}$, and $N_{g}$ are updated at each stage with respect to the newly added generation and transmission infrastructures. To better understand the aforementioned vectors, consider the electricity carrier and all the considered generation options for it in hub $s$. In this hub, if the sets $I_{N \& E}^{G P G}(s, t), I_{N \& E}^{C H P}(s, t)$, and $I_{N \& E}^{G D G}(s, t)$ are comprised of $q_{2}, q_{3}$, and $q_{4}$ members, respectively, the vectors $E$ and $p_{e}$ are defined as follows:

$$
\begin{aligned}
P_{g}^{i n j}(s, b, t)= & \underbrace{\left[n_{I O}^{G P}(1, s, 1, t) \ldots n_{I O}^{G P}(1, s, S, t) \ldots n_{I O}^{G P}\left(q_{1}, s, 1, t\right) \ldots n_{I O}^{G P}\left(q_{1}, s, S, t\right)\right]}_{\mathrm{N}_{g}(s, t)} * \\
& \underbrace{\left[f_{g}(1, s, 1, n, b, t) \ldots f_{g}(1, s, S, n, b, t) \ldots f_{g}\left(q_{1}, s, 1, n, b, t\right) \ldots f_{g}\left(q_{1}, s, S, n, b, t\right)\right]^{\mathrm{T}}}_{\mathrm{F}_{g}(s, b, t)} .
\end{aligned}
$$




$$
\begin{aligned}
E(s, t)= & {\left[n_{I O}^{G P G}(1, s, t) \cdots n_{I O}^{G P G}\left(q_{2}, s, t\right) \cdots\right.} \\
& n_{I O}^{C H P}(1, s, t) \cdots n_{I O}^{C H P}\left(q_{3}, s, t\right) \cdots \\
& \left.n_{I O}^{G D G}(1, s, t) \cdots n_{I O}^{G D G}\left(q_{4}, s, t\right)\right] ; \\
& s=1, . ., S ; t=1, . ., T \\
p_{e}(s, b, t)= & {\left[p^{G P G}(1, s, n, b, t) \ldots p^{G P G}\left(q_{2}, s, n, b, t\right) \ldots\right.} \\
& p^{C H P}(1, s, n, b, t) \ldots p^{C H P}\left(q_{3}, s, n, b, t\right) \ldots \\
& \left.p^{G D G}(1, s, n, b, t) \ldots p^{G D G}\left(q_{4}, s, n, b, t\right)\right] ; \\
& s=1, . ., S ; b=1, . ., B ; t=1, . ., T .
\end{aligned}
$$

Similarly, gas supply and storage systems are the elements of vector $G$. Moreover, the production and withdrawal values are the corresponding elements of $p_{g}$. Consequently, to meet demanded energy forms at each node, the input carriers can be converted into each other by the corresponding mapping matrices as follows:

$$
\begin{aligned}
& L_{e}(s, b, t)-C R(s, b, t)-p^{R E}(s, b, t)=\mathrm{M}_{e}(s, t) \\
& \quad * P_{e}(s, b, t) ; s=1, . ., S ; b=1, . ., B ; t=1, . ., T,
\end{aligned}
$$

$$
\begin{aligned}
& L_{t h}(s, b, t)=\mathrm{M}_{t h}(s, t) * P_{g \rightarrow t h}(s, b, t) \\
& \quad s=1, . ., S ; b=1, . ., B ; t=1, . ., T
\end{aligned}
$$

Since a part of the NG volume injected into each node is consumed in the electricity generation mix of that node, the share of thermal loads in injected volume, $P_{g \rightarrow t h}(s, b, t)$, with regard to the predicted NG demand for that node is given by:

$$
\begin{array}{r}
P_{g \rightarrow t h}(s, b, t)=P_{g}(s, b, t)-\Omega^{\mathrm{G}}(s, b, t)-v^{G S}(s, b, t) \\
-\left(\sum_{i \in I_{N \& E}^{C O}(s, t)} \sum_{n=1}^{n_{I O}^{C O}(i, s, t)} G c(i, s, k, n, b, t)\right. \\
-\sum_{i \in I_{N \& E}^{G P G}(s, t)} \sum_{n=1}^{n_{I O}^{G P G}(i, s, t)} \frac{p^{G P G}(i, s, n, b, t)}{\eta^{G P G}(i)} \\
-\sum_{i \in I_{N \& E}^{C H P}(s, t)} \sum_{n=1}^{n_{I O}^{C H P}(i, s, t)} \frac{p^{C H P}(i, s, n, b, t)}{\eta^{C H P}(i)}
\end{array}
$$

$$
\begin{aligned}
& \left.-\sum_{i \in I_{N \& E}^{G D G}(s, t)} \sum_{n=1}^{n_{I O}^{G D G}(i, s, t)} \frac{p^{G D G}(i, s, n, b, t)}{\eta^{G D G}(i)}\right) \vartheta \\
& s=1, . ., S ; b=1, . ., B ; t=1, . ., T .
\end{aligned}
$$

\section{Numerical analysis}

In this section, the simulation results obtained from two different scenarios conducted on a real large-scale case study are presented and discussed. The scenarios, S1 and S2, are defined as follows; S1: conventional integrated expansion planning and S2: EH-based integrated expansion planning. In S1, expansion of G\&ESs is simultaneously planned regardless of the ability to convert gas and electricity energy carriers into each other to meet heat and electricity demands on the demand side. This state is reached when the rings connecting heat and electricity supply chains to each other on that side are neglected. In doing so, thermal loads are regarded as part of NG demand and thus, some generation/conversion technology options such as GDG and CHP units, gas furnaces, and HPs are removed from candidate options. Through the $\mathrm{S} 2$, the optimal integrated expansion plan of G\&ES is scheduled, while all the considered rings for heat and electricity supply chains are incorporated into the model and the thermal load is excluded from the NG demand.

\subsection{Description of the case study, input data, and assumptions}

To investigate the adequacy of $\mathrm{EH}$ approach in the long-term large-scale ESs' expansion problem, the proposed framework is applied to the Iranian electricity and gas systems, shown in Figure 4. Demand growth rate for each region and capacity factors related to the considered RES generators are also presented in this figure. As can be seen from Figure 4(b), all energy demands are divided between 33 regions. Regardless of energy distribution networks, demands in each node/region are considered as load points. The exchange rate of energy between the regions can increase by enhancing the capacity of existing transmission infrastructures and/or implementing new transmission corridor projects. To incorporate the importance of the gas supply systems fed by common gas fields into the problem, the expansion capacities of gas supply systems related to the uncommon ones are assumed to be restricted. Here, the gas refineries located in region 29 are considered as the supply systems fed by common gas fields. According to the base topology of each under-study ES, candidate transmission corridors, i.e., new transmission lines and/or pipelines, are also depicted in the figure. This context is explored further in Figure 4. 


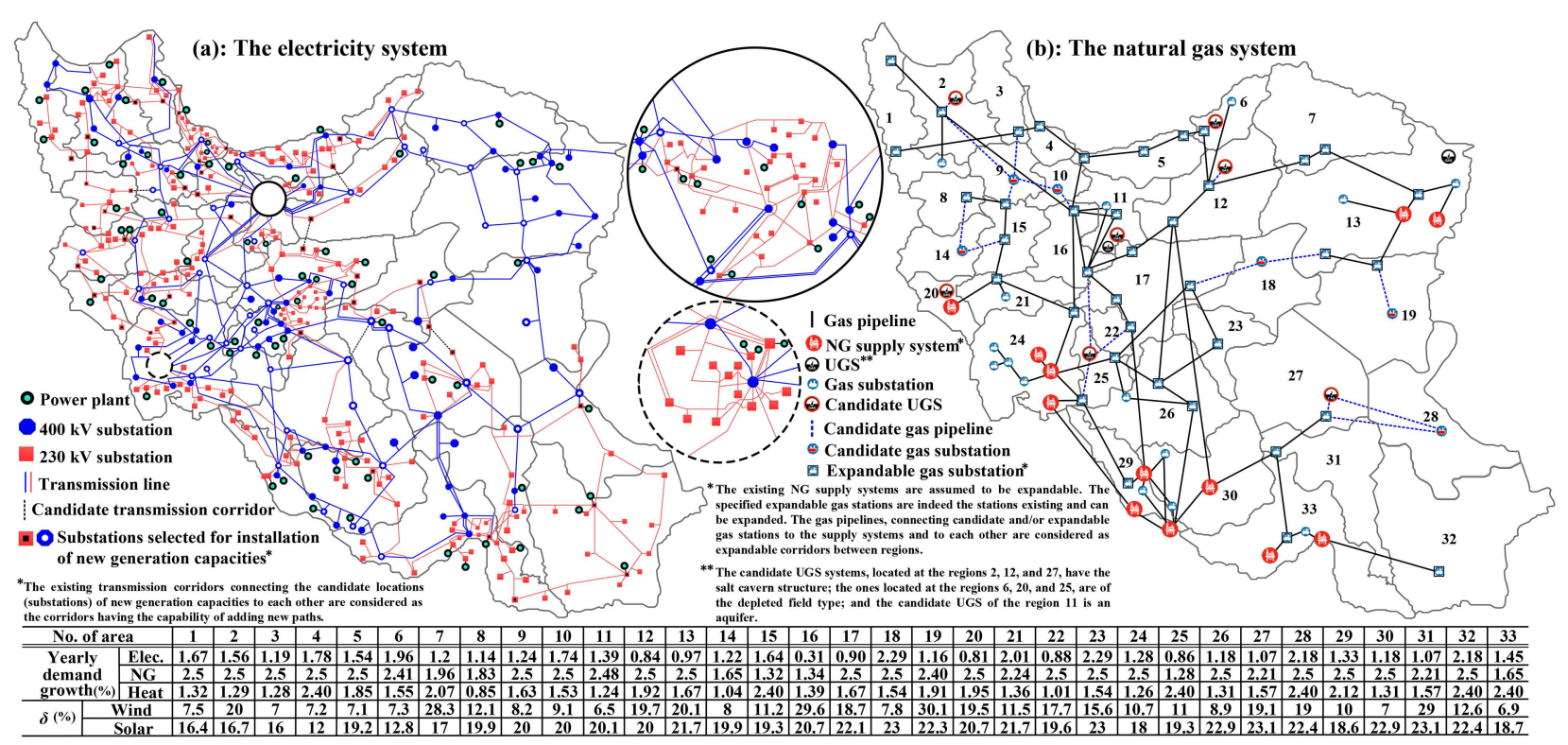

Figure 4. Existing and candidate infrastructures of Iranian power system (a) and NG network (b) with demand and RES capacity factor characteristics corresponding to each region.

The more load blocks the LDC of each carrier has, the more precise the models will be for demand behavior. Hence, each planning stage (year) has been considered here in terms of three load periods, i.e., base, medium, and peak, and each period, in turn, is divided into four monthly load blocks, resulting in twelve blocks. The forecasted number of demands for each carrier at each node during each load block of the year 2020 as the base year of the planning horizon, i.e., 2021-2030, together with the electrical load shedding price, i.e., VOLL, corresponding to each region can be found in [29]. The techno-economic data of candidate infrastructures are itemized in Table 1. In this table, an identification code (ID) is allocated to each technology type of infrastructures in order to facilitate the illustration of simulation results. In Table 1, it should be noted that the polyethylene type of pipelines is starred to be presented in other tables; in the case of storage systems, the first type, denoted by GS1, refers to the above-ground (spherical tank) NG storage system; GS2-GS4 are UGS systems based on aquifer, salt cavern, and depleted field, respectively. The starred operation costs indeed include both operation and O\&M costs. Furthermore, in the aforementioned table, the first three types of gas-fired DG units, i.e., GDG1-GDG3, are from gas turbine technologies; and the last two types, i.e., GDG4 and GDG5, are from reciprocating engine types of gas-firedbased DG technologies. In the case of power generation units, the first three types of considered technologies, i.e., GPG1-GPG3, refer to CCGT units, whereas the last two types, i.e., GPG4 and GPG5, are based on the OCGT technology. Finally, the investment cost of new electricity transmission corridor projects and any additional path for existing topology are also assumed to be $0.24 \mathrm{M} \$ / \mathrm{km}$ and $0.15 \mathrm{M} \$ / \mathrm{km}$, respectively [15].

Formulated as an MILP problem, the scenarios were programmed using the GAMS software and optimized via the CPLEX 11.2.0 solver. The simulations were run on a $\mathrm{PC}$ powered by a Core i3 processor and 3 GB of RAM. Having assumed a cap on the investment budget, i.e., $B^{\text {tot }}=85000 \mathrm{M} \$$, and a $5 \%$ discount rate, the solution results of the conducted scenarios are discussed in the following.

\subsection{Analysis of the co-expansion planning results}

To better highlight the superiority of EH-based expansion planning approach compared with the traditional ESs' co-expansion planning one, the results of the first scenario are presented here. In doing so, how the coordinated planning scheme leads to more optimized expansion strategies in comparison with the separate planning method is also discussed with respect to the available literature [14-21].

As demonstrated by $\mathrm{S} 1$ results summarized in Table 2, the total amount of capacities added to the initial generation mix is $5040 \mathrm{MW}$. Note that in the aforementioned table, the highlighted rows refer to new energy transmission corridors. The NPV of the total cost associated with the added capacities along with installed transmission lines is $4517.91 \mathrm{M} \$$. Feeding the aforementioned added electricity generation capacities together with meeting the gas demand in each region of the case study costs $79856.85 \mathrm{M} \$$ (computed based on the NPV method) for the NG sector. Note that in the co-expansion planning scenario, the operation cost of the newly added GPG units is not taken into account; 
Table 1. Techno-economic data of candidate infrastructures for the gas and electricity energy systems.

\begin{tabular}{|c|c|c|c|c|c|c|c|}
\hline Gas pipeline & ID & GP1 & GP2 & GP3 & GP4 & GP5 & GP6 \\
\hline \multicolumn{2}{|c|}{ Diameter (inch) } & 24 & 30 & 36 & 42 & 48 & 56 \\
\hline \multirow{2}{*}{$\frac{C_{i n v}(\mathrm{M} \$ / \mathrm{km})}{\operatorname{Max} \text { capacity }\left(\mathrm{mm}^{3} / \mathrm{h}\right)}$} & Steel & $\frac{2.9}{0.38}$ & $\frac{4.8}{0.81}$ & $\frac{6.7}{1.25}$ & $\frac{9.4}{168}$ & $\frac{12.2}{2.04}$ & $\frac{12.2}{2.73}$ \\
\hline & Polyeth. & $\frac{2.4}{0.22}$ & $\frac{3.6}{0.54}$ & $\frac{5}{0.86}$ & $\frac{7}{1.41}$ & $\frac{8.5}{1.85}$ & - \\
\hline CHP units & ID & CHP1 & CHP2 & CHP3 & CHP4 & CHP5 & CHP6 \\
\hline \multicolumn{2}{|c|}{ Capacity (MW) } & 0.5 & 1 & 2 & 5 & 10 & 20 \\
\hline \multicolumn{2}{|c|}{$C_{i n v}(\mathrm{M} \$ / \mathrm{MW})$} & 2.1 & 2.08 & 1.96 & 1.85 & 1.8 & 1.5 \\
\hline \multicolumn{2}{|c|}{$C_{o p r}(\$ / \mathrm{MWh})$} & 33 & 32.5 & 32 & 31.7 & 31.1 & 26.9 \\
\hline \multicolumn{2}{|c|}{$C_{O \& M}(\$ / \mathrm{MWh})$} & 12.3 & 11 & 9.81 & 9.44 & 9.25 & 9.1 \\
\hline \multirow{2}{*}{ Effic. $(\%)$} & Gas-Elec. & 35 & 35 & 33 & 32 & 28 & 33 \\
\hline & Gas-Thermal & 44 & 42 & 41.8 & 41 & 40 & 37 \\
\hline NG supply system & ID & SP1 & \multicolumn{2}{|c|}{ SP2 } & SP3 & \multicolumn{2}{|c|}{ SP4 } \\
\hline \multicolumn{2}{|c|}{ Capacity $\left(\mathrm{Mm}^{3} /\right.$ day $)$} & 125 & \multicolumn{2}{|c|}{60} & 20 & \multicolumn{2}{|c|}{10} \\
\hline \multicolumn{2}{|c|}{$C_{i n v}\left(\mathrm{M} \$ / \mathrm{Mm}^{3} /\right.$ day $)$} & 156.95 & \multicolumn{2}{|c|}{160.23} & 164.61 & \multicolumn{2}{|c|}{169.73} \\
\hline \multicolumn{2}{|c|}{$C_{o p r}\left(\$ / \mathrm{Mm}^{3} / \mathrm{h}\right)$} & 57.56 & \multicolumn{2}{|c|}{58.35} & 61.28 & \multicolumn{2}{|c|}{63.48} \\
\hline \multicolumn{2}{|c|}{$\mathrm{C}_{O \& M}\left(\$ / \mathrm{Mm}^{3} / \mathrm{h}\right)$} & 51.67 & \multicolumn{2}{|c|}{43.75} & 30 & \multicolumn{2}{|c|}{12.5} \\
\hline Gas reservoirs & ID & GS1 & & & GS3 & & \\
\hline Capacity (1 & $\left.n^{3}\right)$ & 0.015 & 10 & $15 \%$ & $300_{ \pm 15 \%}$ & 1000 & $15 \%$ \\
\hline$C_{i n v}(\mathrm{M} \$ /$ & $\left.m^{3}\right)$ & 1.03 & & & 0.781 & & \\
\hline$\mu \& \lambda(\$ / \mathrm{Mr}$ & $/ \mathrm{h})$ & 1200 & & & 5000 & & \\
\hline $\mathrm{C}_{O \& M}(\$ / \mathrm{M}$ & $3 / \mathrm{h})$ & 250 & & & 1150 & & \\
\hline Gas compressors & ID & $\mathrm{CO} 1$ & & & $\mathrm{CO} 3$ & & \\
\hline Capacity ( & W) & 25 & & & 75 & & \\
\hline Trans. rate $(1$ & $\left.n^{3} / h\right)$ & 1.24 & & & 4.58 & & \\
\hline$C_{i n v}(\mathrm{M} \$ /$ & W) & 2 & & & 3.86 & & \\
\hline$C_{o p r}^{*}(\$ / \mathrm{Mn}$ & $/ \mathrm{h})$ & 41 & & & 38.2 & & \\
\hline Heat pumps & ID & HP1 & & & HP3 & & \\
\hline Capacity ( & W) & 10 & & & 30 & & \\
\hline Perform. coe & cient & 3.1 & & & 3.15 & & \\
\hline$C_{i n v}(\mathrm{M} \$ /$ & W) & 0.91 & & & 0.88 & & \\
\hline$C_{o p r}^{*}(\$ / \mathrm{M}$ & & 252.6 & & & 248.3 & & \\
\hline Gas furnaces & ID & FU1 & & & FU3 & & \\
\hline Capacity (MN & tu $\mathrm{u} / \mathrm{h})$ & 40 & & & 60 & & \\
\hline$C_{i n v}(\mathrm{M} \$ / \mathrm{M}$ & Btu) & 0.045 & & & 0.039 & & \\
\hline$C_{o p r}^{*}(\$ / \mathrm{MM}$ & $\mathrm{u} / \mathrm{h})$ & 3.1 & & & 3.9 & & \\
\hline Effic. $(9$ & & 90 & & & 85 & & \\
\hline GDG units & ID & GDG1 & & & GDG3 & GDG4 & GDG 5 \\
\hline Capacity ( & W) & 10 & & & 40 & 2 & 3 \\
\hline$C_{i n v}(\mathrm{M} \$ /$ & W) & 1.3 & & & 0.82 & 1.8 & 1.7 \\
\hline$C_{o p r}(\$ / \mathrm{M}$ & & 35.5 & & & 25 & 26.9 & 25.3 \\
\hline $\mathrm{C}_{O \& M}(\$ / \mathrm{I}$ & $N \mathrm{~h})$ & 13 & & & 7 & 11.3 & 10 \\
\hline Effic. ( & & 25 & & & 35.5 & 33 & 35 \\
\hline GPG units & ID & GPG1 & & & GPG 3 & GPG 4 & GPG5 \\
\hline Capacity ( & W) & 80 & & & 160 & 160 & 200 \\
\hline$C_{i n v}(\mathrm{M} \$ /$ & W) & 1 & & & 0.96 & 0.83 & 0.81 \\
\hline$C_{o p r}(\$ / \mathrm{M}$ & & 16.1 & & & 14.5 & 21.6 & 21.6 \\
\hline $\mathrm{C}_{O \& M}(\$ / \mathrm{I}$ & $\mathrm{Nh})$ & 43 & & & 38 & 159 & 156 \\
\hline Effic. $(?$ & & 55 & & & 61 & 41 & 41 \\
\hline
\end{tabular}


Table 2. The details of S1 expansion results.

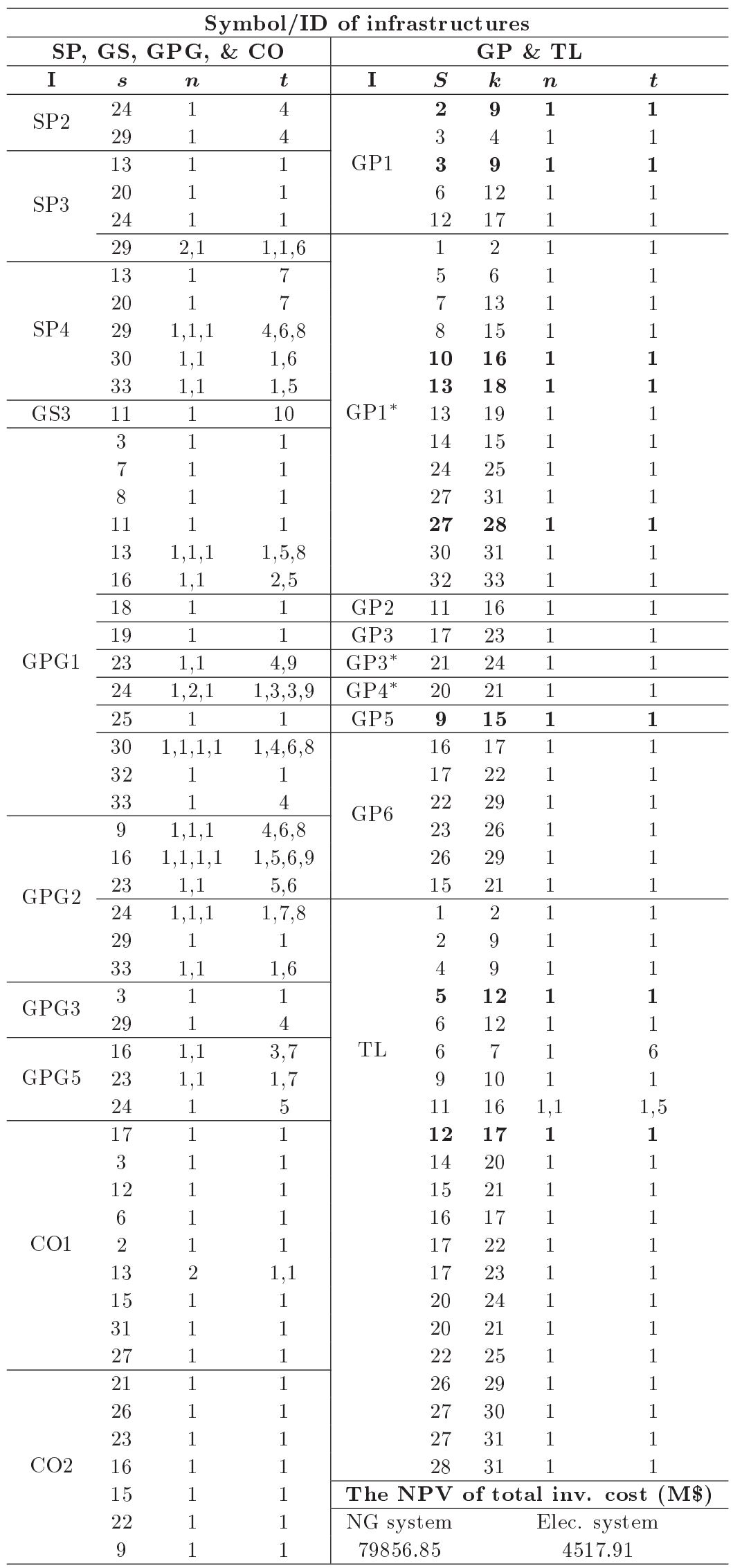


Table 3. IPPs share and the amount of EENS in S1.

\begin{tabular}{ccccccc}
\hline & \multicolumn{2}{c}{ EENS } & \multicolumn{4}{c}{ IPPs } \\
\cline { 2 - 6 } $\boldsymbol{s}$ & $\boldsymbol{t}$ & $\mathbf{M W h}$ & $\boldsymbol{i}$ & $\boldsymbol{n}$ & $\boldsymbol{t}$ & $\mathbf{M W}$ \\
\hline 7 & - & 0 & Wind & 1 & 8 & 15 \\
12 & 10 & 45761 & - & 0 & - & 0 \\
27 & - & 0 & Solar & 1 & 7 & 50 \\
28 & 9 & 2157 & Solar & 1 & 8 & 50 \\
28 & 10 & 20683 & & & & \\
\hline \multicolumn{2}{r}{ Total } & 68601 & & & & 115 \\
\hline
\end{tabular}

this cost indeed results from the operation cost of gas supply systems.

Table 3 shows the details of IPPs contribution and the amounts of regions' EENS during the planning horizon. The NPV of total incentives allocated to the RES-based generators granting FIT of $105 \$ / \mathrm{MWh}$ and 122.5\$/MWh for wind and solar types [30], respectively, is $60.687 \mathrm{M} \$$. In this context, as can be seen in Table 3 and Figure 4, the regions characterized by lower electricity demand growth and higher $\delta$ have been more attractive options for attracting IPP contribution. Nevertheless, the share of RES in meeting the growing demand is negligible, which may be derived from low VOLL or incentives paid to the renewable generators. It is noteworthy that in the proposed model, the uncertainties associated with operation of the RES-based units are addressed by assuming reasonable values for their utilization hours per year, i.e., 1700 and 1400 hour/year, corresponding to the wind and solar technologies, respectively [31]. By using the S1, the total amount of EENS and the NPV of the corresponding VOLLs are $68601 \mathrm{MWh}$ and $67.072 \mathrm{M} \$$, respectively. The differences in the amounts of EENS for different regions are derived from the location of GPG units, the profitability of RES-based units for the IPPs, the characteristics of transmission lines, and above all, defined prices for the shed loads.

As demonstrated by a wide range of research works focusing on integrated expansion/operation planning of G\&ES [14-21,32,33], the co-planning method reaches more optimal expansion/operation strategies for both G\&ESs from a general point of view compared to a situation where the strategies of the aforementioned ESs are separately planned. In the case of co-expansion planning studies, what makes simultaneous expansion strategies more optimal is the improvement of the utilization efficiency of ES infrastructure, which in turn is the reflection of incorporating the interactions existing between G\&ESs into the planning. This can be better understood by the evaluation of the impacts that electricity expansion plans can have on the NG sector in the co-expansion planning approach and the separate expansion planning method, while the electricity sector can be treated as one of the main NG consumers. As a matter of fact, according to the available literature [14-21], new capacities added to electricity generation mixes through separate expansion planning studies are only translated into a part of the projected NG demand with a predefined and fixed location for the NG sector, whereas in the co-expansion approach, similar to S1 simulated here, variability of the location and capacity of NG-based generation options creates a degree of flexibility for the NG sector and, consequently, a compromise between gas and electricity networks in terms of expansion, resulting in achieving more optimal expansion strategies. Accordingly, GPG units, like a support put under a rod, can make an optimal balance between the lengths of pipelines and transmission lines.

In todays' large-scale gas ESs, the effectiveness of the role of GSSs in optimizing the size of gas supply systems and pipelines has been proven, as outlined in $[14,17,18,20]$. Here, in order to evaluate the performance of GSS added to the under-study gas system in region 11, it is necessary to ass the NG load profile of this region, the gas flow rate of new gas pipeline, feeding of the relevant gas demand in that region, and the generation behavior of relevant gas supply systems. In this regard, the NG load profile of region 11, the charging behavior of the GSS added to the aforementioned region, the process of stored gas volume changes, and the gas flow rate of 11-16 pipelines, as the only corridor covering NG demand growth of region 11, are depicted in Figure 5. The NG load profile of region 11 is indeed the sum of NG demand, including gas and heat demands, and the amount of gas consumed by the electricity generation capacity added to the relevant generation mix.

Regarding the arrangement of expanded gas pipelines and implemented transmission line projects summarized in Table 1, it can be observed that the gas and electricity demands of region 11 along with regions $5,6,10,12,16,17,22,23,26$, and 29 are fed by the gas supply systems added to region 29 . To better appreciate the dispatch of supplied gas in region 29 , the implemented gas pipeline projects are shown in Figure 6. From this figure, it is simply found that the pipeline $16-11$ feeding region 11 is originated from the gas supply systems in region 29 . Note that the electricity demands of all the above-mentioned regions, except region 6 , are met by the generation capacities added to regions 16,23 , and 29, which in turn are fed by the gas supply systems of region 29 . To investigate the impact that the GSS may have onto the gas supply systems, their generation behavior and the sum of total NG demands of all the regions covered by them during the operation period of the added GSS are given in Figure 7. 


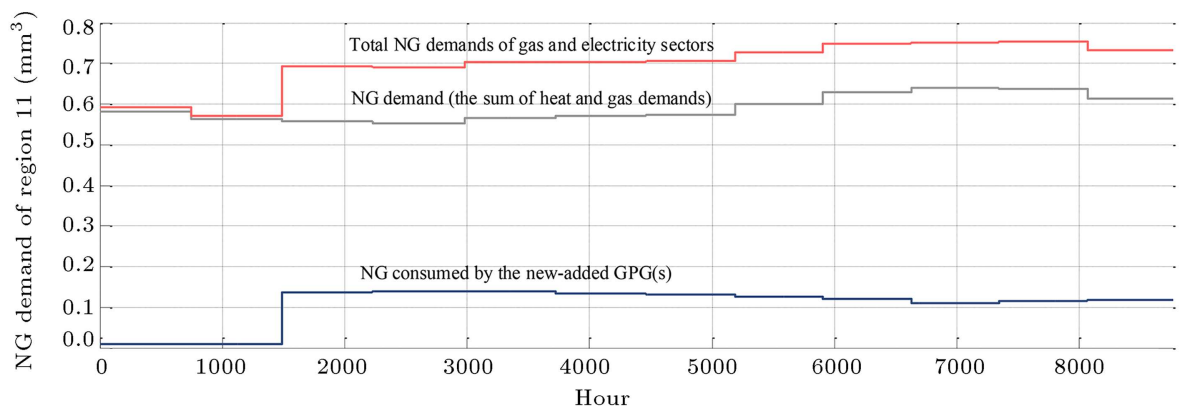

(a)

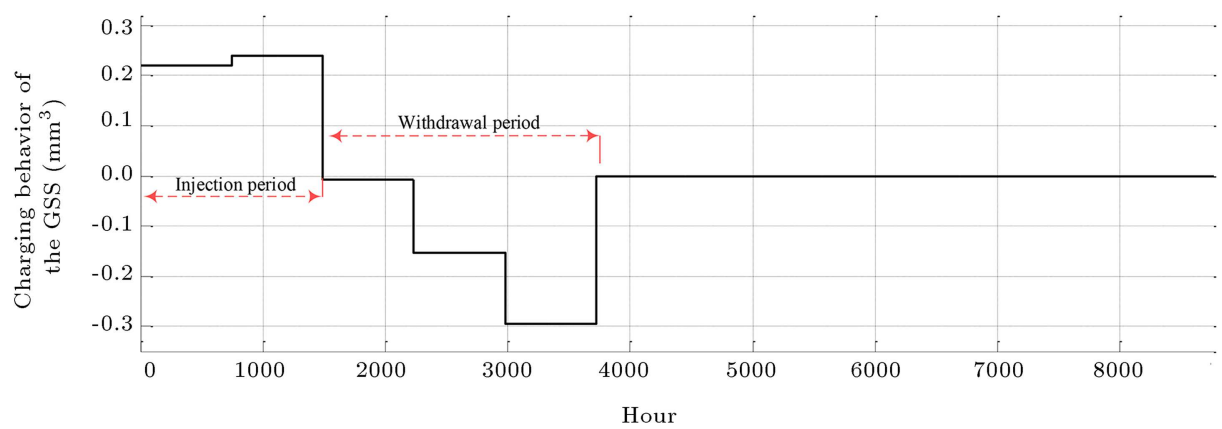

(b)

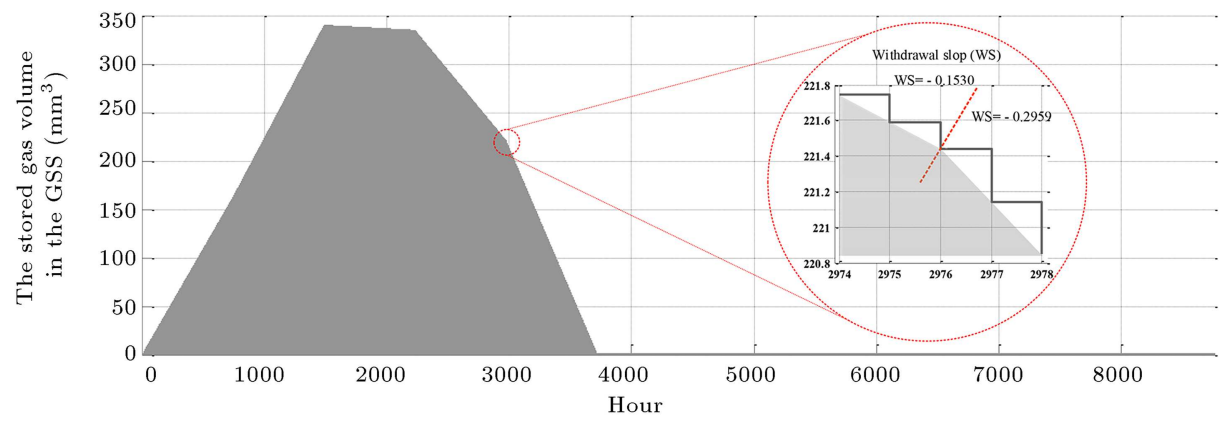

(c)

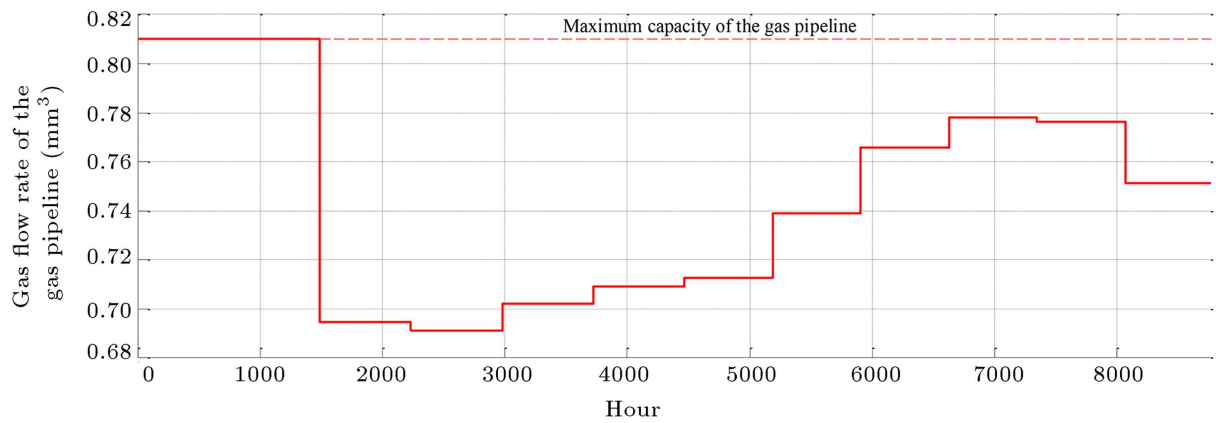

(d)

Figure 5. (a) Total NG demands (sum of gas and electricity NG demands) of region 11. (b) Injection (charge) and withdrawal (discharge) amounts of the GSS. (c) The volume changes of gas stored in the GSS. (d) The flow rate of gas pipeline 16-11 during the last planning stage (in-operation year of GSS).

The trade-off made between gas and electricity sectors in meeting total gas demands (the sum of gas and electricity demands) of the regions covered by the SP added to region 29 is apparent in Figure 5. As can be seen from this figure, by adding the GSS to region 11, the consumed gas by the added generation mix, i.e., the $80-\mathrm{MW}$ CCGT unit, to this region is reduced to the lowest possible level during the first months of GSS in the operation year, i.e., two first months of the last planning year. During this period, total electrical load of the aforementioned region is met by the generation mix of the adjacent region, i.e., region 


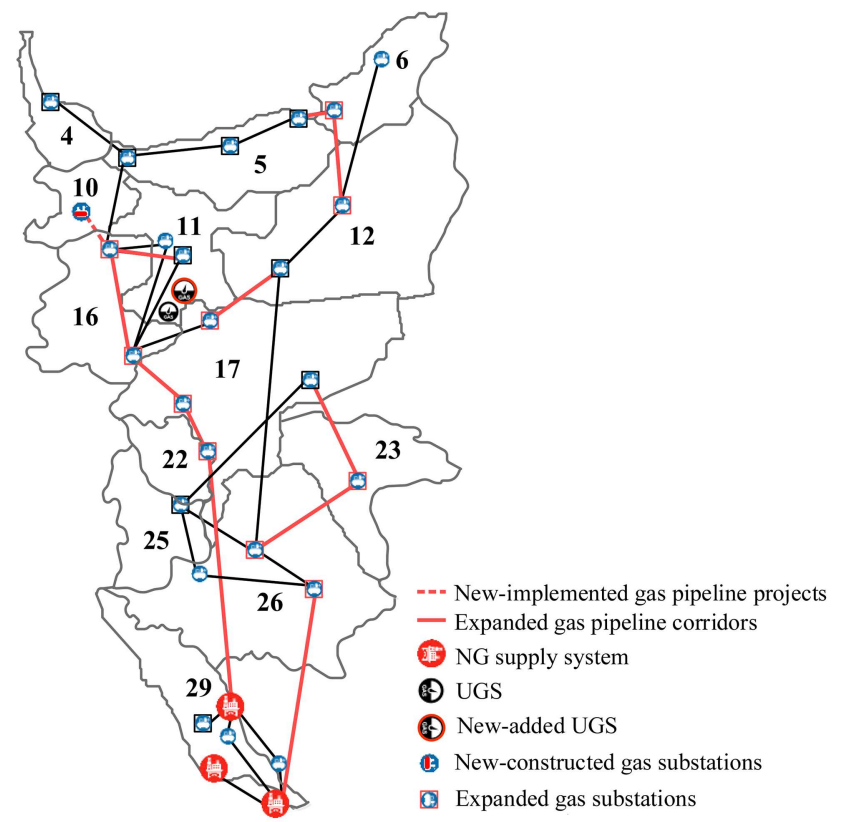

Figure 6. The sketch of NG demand nodes covered by the gas supply systems of region 29 .

16. At the same time, as observable from Figure 5(d), the NG volume delivered to region 11 by pipeline 1611 is at the highest possible level with respect to the pipeline capacity limit. Reducing the gas consumption of the electricity generation mix and receiving the highest possible NG volume through the pipeline provide the possibility of storing NG in the GSS in region 11 so that at the end of the aforementioned period, the stored gas volume in the GSS reaches $340 \mathrm{~mm}^{3}$. At this time, indeed, the GSS has been fully charged. Following the increase in electrical loads of the regions and in the gas demands of the generation mixes, as can be seen from Figure 7, the sum of total NG loads of the regions is greater than the maximum installed capacity of $\mathrm{SP}$ in region 29 , i.e., $6.25 \mathrm{~mm}^{3} / \mathrm{h}(150$ $\mathrm{mm}^{3} /$ day), during the peak period. This imbalance between NG supply capacity and level of demand is indeed derived from the presence of GSS in region 11. In Figures 5(b) and 7(a), the GSS could prevent greater investment in the gas supply systems by meeting a big share of peak NG demand during the withdrawal period in accordance with the total NG demand profile, shown in Figure 7(b). Obviously, participation of the GSS in load meeting can lead to better budget allocation and assets management. Another notable point about the above-discussed trade-off between gas and electricity generation and demand nodes relates to the shed load in region 12 during the monitored operation period. According to Figure 7, the capacity limit of the GSS (or of the relevant feeding gas pipeline) is the reason why the whole NG demand cannot be met by the GSS during the end hours of the peak period at the planned capacity for the SP of region 29. In other words, the comparison between the NG generation level and demand in Figure 7, when the level of the stored

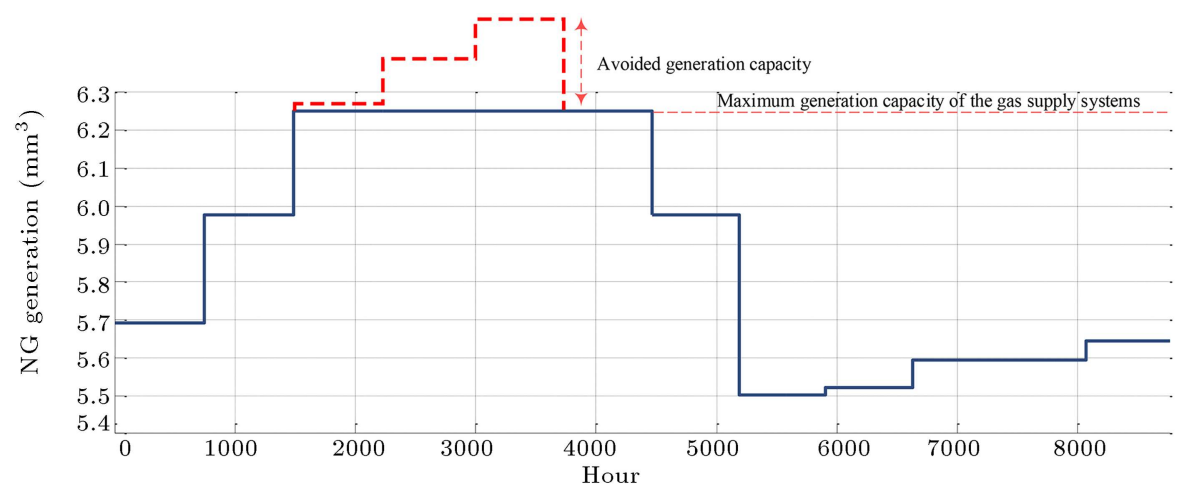

(a)

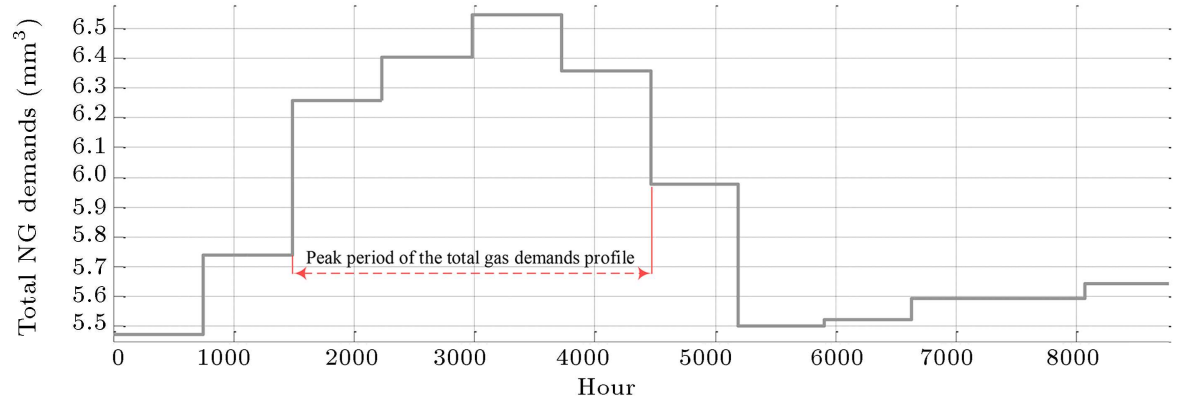

(b)

Figure 7. (a) Generation behavior of the gas supply systems in region 29 and (b) the sum of NG demands of the regions covered by the gas supply systems of region 29 during the last planning stage (in-operation year of GSS). 
NG volume in GSS reaches its own minimum amount, demonstrates that the GSS cannot completely cover the capacity shortage of the gas supply systems of region 29. This mismatch was reflected in electrical load shedding in region 12 , which might have the lowest VOLL among other regions supplied by the new SP installed in region 29 .

\subsection{Analysis of the EH-based co-expansion planning results}

Main findings of the built framework in this work are analyzed here. As can be inferred from the details provided by Table 4 for S2, the focus on supplying energy demands varies slightly from the capacity expansion of basic ES infrastructure to the employment of smallscale generation and/or conversion technologies in the planned expansion strategy. It is noteworthy that in this scenario, similar to S1, the operation costs of NGbased electricity generation technologies lie in the costs associated with gas supply systems; hence, they are excluded from the OF. Note that in the aforementioned table, the highlighted rows refer to the new energy transmission corridors.

In the case of the provided details in Table 4, at first glance, it may seem that considerable investment in the new heat and electricity supply chain rings including CHP, HPs, and GDG units through the second scenario has yielded considerable costs, compared to S1. Despite the plurality and diversity of the added technologies and infrastructures through S2, the NPV of total costs of both G\&ESs under study in this scenario equals $83721.54 \mathrm{M} \$$. This cost, together with other expenditures derived from shed loads and IPPS participation, is $772.87 \mathrm{M} \$$ lower than the total costs of S1. Analysis of the findings indicates that different changes to the expansion strategy affected by higher flexibility provided in load meeting and infrastructures characterized by higher level of EE could result in cost saving. Obviously, the source of changes has been the employment of the EH concept. It should be noted that through the second scenario, all new gas pipelines and transmission lines have been added to the base topology of the studied G\&ESs through the first planning stage (Year 1).

From the viewpoint of flexibility, excluding the heat loads from the total NG demand of each region together with more completely modelling the loads' supply chains can lead to decrease in required investment costs of the basic infrastructures of both G\&ESs. In other words, the reductions in the capacity electricity generation and gas and electricity transmission infrastructure are, in fact, the reflection of investing in the candidate distributed generation/conversion technology options and of separating final forms of energy demanded. Under these circumstances, employing these generators/convertors in some cases can lead to relatively less cost than the expansion of basic ES infrastructure to meet the projected demands. For instance, in the case of gas pipelines capacity, as can be seen from the summarized results in Table 4, incorporation of the HPs role into the under-study supply chains can remarkably reduce the diameter of required gas pipeline projects. As a matter of fact, the aforementioned technologies and CHP units provide the possibility of supplying heat loads consisting of a big part of NG demands by using both G\&ES infrastructures. Furthermore, the heat demand can only be met by the pipelines in terms of NG demand. By contrast, when the gas and heat demands are considered separately in accordance with what exists in the real world, the NG demand in each region has the opportunity to be supplied by the pipelines characterized by lower capacity (diameter) if the relevant heat load meeting via the electricity carrier (HPs) is more cost effective. This subject can be better appreciated in case of the regions with a higher heat demand growth rate, e.g., region 4 (see Figure 4). In this energy hub, through $\mathrm{S} 1$, the $\mathrm{NG}$ demand encompassing the heat demand is met by the GP1type gas pipeline (gas pipelines 9-3 and 3-4) which costs $0.38 \mathrm{M} \$ / \mathrm{km}$ (see Table 1 ). However, through $\mathrm{S} 2$, the GP1* type of candidate technologies with lower capacity and investment cost has been selected for the aforementioned gas pipeline path, resulting in $0.5 \mathrm{M} \$$ cost saving for each kilometer of that path. This reduction in the costs is indeed the reflection of HPs utilization in region 4 for meeting heat demand with the aid of generated power in region 9 (see Table 4). Note that the difference in the expansion plan of S1 and S2 for the gas system under study originates from the pipeline capacity (diameter), while the new topology of NG network and total length of constructed pipelines in both scenarios are almost the same.

From the viewpoint of EE, incorporation of highly efficient generation/conversion technologies, such as CHP units, into the supply chain models of energy carriers demanded by end-users can obviously provide cost saving opportunities for ES planners. Here, to better appreciate this context, the planned expansion strategies are compared through both scenarios for meeting all demands of region 32 . The peak levels of heat, gas, and electricity in this region during the last planning stage are $2.27^{*} 10^{-6}$ MMBtu (equal to $6.648^{*} 10^{-5} \mathrm{~mm}^{3} / \mathrm{h}$ gas), $6.22^{*} 10^{-4} \mathrm{~mm}^{3} / \mathrm{h}$, and $46.47 \mathrm{MWh}$ (equal to $80.15^{*} 10^{-3} \mathrm{~mm}^{3} / \mathrm{h}$ gas), respectively [29]. As can be seen from the result tables, i.e., Tables 2 and 4, through both of the simulated scenarios, the gas pipeline with minimum transmission rate among exiting technology options is employed to meet the relevant $\mathrm{NG}$ demands. In S1, the delivered gas to the aforementioned region is consumed in both gas and electricity sectors for meeting the NG 
Table 4. The details of S2 expansion results.

\begin{tabular}{|c|c|c|c|c|c|c|c|c|c|c|}
\hline \multicolumn{11}{|c|}{ Symbol / ID of infrastructures } \\
\hline \multicolumn{4}{|c|}{ SP, GS, GPG, \& CO } & \multicolumn{4}{|c|}{ CHP \& HP } & \multicolumn{3}{|c|}{$\mathbf{G P}^{*} \& \mathbf{T L}^{*}$} \\
\hline$i$ & $s$ & $n$ & $t$ & $i$ & $s$ & $n$ & $t$ & $i$ & $s$ & $k$ \\
\hline \multirow{2}{*}{$\mathrm{SP} 2$} & 24 & 1 & 4 & \multirow{5}{*}{$\mathrm{CO} 1$} & 16 & 1 & 1 & GP1 & 11 & 16 \\
\hline & 29 & 1 & 4 & & 27 & 1 & 1 & \multirow{18}{*}{ GP $1 *$} & 1 & 2 \\
\hline \multirow{4}{*}{ SP3 } & 13 & 1 & 1 & & 23 & 1 & 1 & & 2 & 9 \\
\hline & 20 & 1 & 1 & & 24 & 1 & 1 & & 3 & 9 \\
\hline & 24 & 1 & 1 & & 31 & 1 & 1 & & 3 & 4 \\
\hline & 29 & 2,1 & $1,1,6$ & \multirow{5}{*}{$\mathrm{CO} 2$} & 9 & 1 & 1 & & 5 & 6 \\
\hline \multirow{5}{*}{$\mathrm{SP} 4$} & 13 & 1 & 7 & & 15 & 1 & 1 & & 6 & 12 \\
\hline & 20 & 1 & 7 & & 21 & 1 & 1 & & 7 & 13 \\
\hline & 29 & $1,1,1,1$ & $4,6,8,9$ & & 22 & 1 & 1 & & 8 & 15 \\
\hline & 30 & 1,1 & 1,6 & & 26 & 1 & 1 & & 10 & 16 \\
\hline & 33 & 1,1 & 1,5 & \multirow{5}{*}{ CHP1 } & 18 & 1 & 7 & & 12 & 17 \\
\hline \multirow{8}{*}{ GPG1 } & 9 & 1,1 & 4,8 & & 20 & 1 & 9 & & 13 & 18 \\
\hline & 13 & 1,1 & 1,6 & & 24 & 1 & 9 & & 13 & 19 \\
\hline & 16 & 1,1 & 8,10 & & 28 & 1 & 1 & & 14 & 15 \\
\hline & 24 & 1 & 4 & & 30 & 1 & 7 & & 24 & 25 \\
\hline & 25 & 1 & 1 & \multirow{4}{*}{ CHP2 } & 8 & 1 & 8 & & 27 & 31 \\
\hline & 29 & $1,1,1$ & $1,3,7$ & & 18 & 1 & 1 & & 27 & 28 \\
\hline & 30 & 1 & 1 & & 19 & 1 & 9 & & 30 & 31 \\
\hline & 33 & 1,1 & 1,4 & & 29 & 1 & 1 & & 32 & 33 \\
\hline & 9 & 1 & 3 & & 7 & 1 & 1 & GP2 & 17 & 23 \\
\hline$G_{P} G_{2}$ & 16 & $1,1,1$ & $1,2,9$ & & 19 & 1,1 & 1,5 & GP3 & 20 & 21 \\
\hline प1 U2 & 24 & 1 & 3 & & 20 & 1 & 1 & GP3* & 21 & 24 \\
\hline & 33 & 1 & 6 & CHP3 & 23 & 1,1 & 6,8 & GP5 & 9 & 15 \\
\hline GPG3 & 23 & $1,1,1$ & $4,5,8$ & & 27 & 1 & 1 & & 15 & 21 \\
\hline GPG4 & 30 & 1 & 4 & & 30 & 1 & 1 & & 16 & 17 \\
\hline & 9 & 1,1 & 1,5 & & 31 & 1 & 1 & GP6 & 17 & 22 \\
\hline GPG5 & 16 & $1,1,1$ & $3,5,6$ & & 7 & 1 & 1 & & 22 & 29 \\
\hline & 23 & 1,1 & 1,7 & & 8 & 1 & 1 & & 23 & 26 \\
\hline & 24 & $1,1,1$ & $1,5,8$ & & 13 & 1 & 10 & & 26 & 29 \\
\hline & 7 & 1,1 & 1,5 & CHP 4 & 23 & 1 & 1 & & 1 & 2 \\
\hline & 8 & $1,1,1$ & $1,4,7$ & & 24 & 1 & 1 & & 2 & 9 \\
\hline & 18 & $1,1,1$ & $5,7,10$ & & 25 & 1 & 1 & & 2 & 3 \\
\hline GDG1 & 19 & 1,1 & 1,5 & & 27 & 1 & 1 & & 4 & 9 \\
\hline Hit & 20 & 1 & 1 & & 3 & 1 & 1 & & 5 & 12 \\
\hline & 29 & 1 & 9 & & 9 & 1 & 1 & & 6 & 12 \\
\hline & 31 & $1,1,1$ & $1,3,6$ & CHP5 & 10 & 1 & 1 & & 9 & 10 \\
\hline & 32 & 1 & 7 & & 32 & 1 & 1 & & 12 & 17 \\
\hline & 23 & 1 & 10 & & 33 & 1 & 1 & & 14 & 20 \\
\hline & 24 & 1 & 10 & CHP6 & 13 & $1,1,1$ & $1,4,6$ & TL & 15 & 21 \\
\hline GDG2 & 33 & 1 & 10 & & 1 & $1,1,1$ & $1,4,7$ & & 16 & 17 \\
\hline & 9 & 1 & 10 & & 2 & $1,1,1,1$ & $1,3,6,9$ & & 17 & 22 \\
\hline & 32 & 1 & 3 & & 4 & $1,1,1$ & $1,6,9$ & & 17 & 23 \\
\hline & 18 & 1 & 1 & & 5 & $1,1,1$ & $1,3,5$ & & 20 & 21 \\
\hline GDG3 & 24 & 1 & 10 & & 6 & 1,1 & 1,6 & & 20 & 24 \\
\hline & 29 & 1 & 9 & & 12 & 1 & 1 & & 22 & 25 \\
\hline & 30 & 1,1 & 8,9 & HP1 & 14 & 1 & 1 & & 26 & 29 \\
\hline & 7 & 1 & 10 & & 15 & $1,1,1,1$ & $1,3,6,9$ & & 27 & 30 \\
\hline & 18 & 1 & 9 & & 16 & 1,1 & 1,7 & & 27 & 31 \\
\hline GDG4 & 19 & 1 & 10 & & 17 & 1,1 & 4,5 & & 28 & 31 \\
\hline & 31 & 1 & 9 & & 21 & 1,1 & 1,7 & The NPV & f total i & \\
\hline & 31 & 1 & 9 & & 22 & 1 & 1 & of the su & ly chaiı & \\
\hline GDG5 & 20 & 1 & 8 & & 26 & 1,1 & 1,6 & NG system & Elec. sy & \\
\hline & 2 & 1 & 1 & & 4 & 1 & 3 & 78895.61 & 4153. & \\
\hline & 3 & 1 & 1 & $\mathrm{HP} 2$ & 5 & 1 & 7 & The NPV & f total i & \\
\hline & 6 & 1 & 1 & & 11 & 1 & 10 & of the & necting & \\
\hline $\mathrm{CO} 1$ & 12 & 1 & 1 & & 17 & 1,1 & 1,7 & & $(\mathrm{M} \$)$ & \\
\hline & 13 & 2 & 1,1 & HP3 & 11 & 1 & 4 & CHP units & Heat pu & \\
\hline & 15 & 1 & 1 & HP 4 & 11 & 1,1 & 1,6 & 261.58 & 410.8 & \\
\hline
\end{tabular}


demand and feeding the newly added 80-MW GPG unit, respectively. In $\mathrm{S} 2$, separation of heat and $\mathrm{NG}$ demand from each other as well as consideration of the conversion ability of the demanded energy carriers to each other as a result of completing the models of supply chains under study in the EH context prevent investment in new generation capacity. Under the new circumstances of the supply chain model, in fact, the combination CHP and GDG units is planned to meet heat and electricity demands, thus avoiding high investment cost in generation mix and decreasing NG sector operation cost. The latter is derived from replacing basic infrastructures, such as power plants mostly characterized by low efficiencies, with highly efficient small-scale generation options.

In the case of the expansion strategies obtained from conducted scenarios, it can be seen that utilization of HP and CHP units has been more attractive than gas furnace technologies to meet the heat demand. This may be derived from the high coefficient of performance in HPs and the large difference between capital cost of G\&ES infrastructures. As expansion costs of NG systems are very greater than the costs of electricity systems, utilization of FU with NG carrier input for meeting heat demands does not seem more economically.

In the second scenario, the amounts of unserved load are 803.52 MWh and 193.44 MWh related to regions 18 and 19 at the ninth planning stage, respectively. The total NPV of the shed loads is $1.112 \mathrm{M} \$$. By comparing the total EENS amounts of two scenarios, it can be inferred that the presence of distributed generations in the $\mathrm{EH}$ framework can remarkably reduce load shedding by increasing load meeting options. In S2, no IPPs contribute to meeting electricity load. The flexibility in meeting load along with the lack of emission cap in the model can be treated as the reasons for this part of S2 expansion strategy.

\section{Concluding remarks}

This paper devised an analytical framework to assess the effectiveness of Energy $\mathrm{Hub}(\mathrm{EH})$ approach in co-expansion planning of Gas and Electricity System (G\&ES) with the necessity of enhancing EE, attenuating the energy sector impact on climate change, and modifying traditional planning methods to determine more optimal and compatible strategies in ESs' planning problems. In this regard, after scrutinizing the nature of this approach based on the supply chain concept and reviewing the chain of events that increased the dependency of G\&ESs, a suitable MILP-based model for integrated planning of the aforementioned ESs was presented. According to the pursued objective in the proposed framework, two planning scenarios were conducted and optimal expansion strategies obtained from the simulation of each scenario were discussed in depth. The following highlights the main conclusions drawn out of the pursued framework:

1. The EH approach is not a new-invented planning tool for different ESs, although it is a new look at their co-planning manners. Thus, the concept of 'multi-carrier energy systems' is not a new system among energy systems, but is a new attitude to integrated planning of ESs;

2. Regardless of the EH concept, the traditional expansion co-planning of G\&ES results in more optimal expansion plans than separate planning of these ESs, as outlined in the literature. Moreover, given that the electricity demand will be finally a part of NG demand, it can be derived from the variable state of the Gas-fired Power Plants (GPG) units' locations, making the problem more flexible and consequently, providing a trade-off between the lengths of gas and electricity transmission lines;

3. In the next stage, EH-based expansion co-planning of G\&ES and, as a consequence, considering the conversion ability for final forms of demanded energy carriers offer more options for supplying the loads, thus increasing flexibility level of the problem once again. This finding results in achieving more optimal and realistic expansion strategies than the conventional integrated planning method;

4. Gas storage systems can play a prominent role in balancing energy production and demands and in postponing investment costs of NG system expansion capacity.

Regarding the nature of $\mathrm{EH}$ approach and the importance of adopting a comprehensive view on ESs in co-planning problems, the present work established a planning framework in the traditional (vertically integrated) structure of the ESs. Obviously, the application of the $\mathrm{EH}$ approach to the liberalized structure of ESs would be very sophisticated from the mathematical perspective, affected by the need for considering relevant energy markets associated with the data exchange constraints as well as the conflicts that may exist between independent operators of the systems. Future research should propose an expansion co-planning model applicable to G\&ESs associated with their markets in a liberalized environment.

\section{Nomenclature}

\section{Indices}

$i, s, n, b, t \quad$ Index corresponding to an infrastructure type, installation area, number of infrastructures, loads-block, and a planning horizon stage, respectively 
$S, B, T \quad$ Index corresponding to the total number of areas, loads-blocks, and planning horizon intervals, respectively

\section{Superscripts}

\section{$S P \quad$ Gas supply systems}

GP Gas Pipelines

GS Gas Storage systems

$F U \quad$ Gas furnaces

$\mathrm{CO} \quad$ Gas compressors

HP Heat Pumps

$G P G \quad$ Gas-fired Power Plants

CHP Combined Heat and Power units

GDG Gas-fired Distributed Generation units

$T L$

$R E$ Transmission Lines

\section{Constants/parameters}

$C_{\text {inv }} \quad$ Investment cost

$C_{O \& M} \quad$ Fixed operation and maintenance cost

$C_{\text {opr }}$ Variable operation cost

$C_{\text {inc }}$

$\mu, \lambda \quad$ Injection and withdrawal cost of gas

Renewables' incentive-based support scheme cost storage systems, respectively

$\bar{v}, \underline{v} \quad$ Maximum and minimum bounds of gas storage systems, respectively

$\bar{v}^{i n j}, \underline{v}^{i n j} \quad$ Maximum and minimum injection bounds of gas storage systems, respectively

$\bar{v}^{\text {with }}, \underline{v}^{\text {with }}$ Maximum and minimum withdrawal bounds of gas storage systems, respectively

$\bar{p}, \underline{p} \quad$ Maximum and minimum power generation capacity, respectively

$\bar{g}, \underline{g} \quad$ Maximum and minimum gas production capacities of gas supply systems, respectively

$\bar{h}, \underline{h} \quad$ Maximum and minimum heat power generation capacities, respectively

$\delta \quad$ Renewable-based distributed generation capacity factor multiplied by 8760

$\vartheta \quad$ Electricity-to-gas conversion factor

$\bar{N} \quad$ Maximum number of an infrastructure that can be installed during the planning horizon

$\bar{f}_{g} \quad$ Maximum flow capacity of a pipeline

$\tau \quad$ Seasonal storage factor.

$r \quad$ Discount year.

$$
\begin{aligned}
& \rho^{\text {out }}, \rho^{\text {in }} \\
& \varphi
\end{aligned}
$$

$h p$

$\xi$

$\xi_{g}$

$\eta$

Pc

Gc

$B^{\text {tot }}$

$\overline{E E N S}$

VOLL

$\Phi^{E}, \Phi^{H}$

$\Omega^{G}, \Phi^{G}$

$\Phi^{G} \Phi^{\bar{G}}$

$\Omega^{E}, \Omega^{\bar{G}}$

\section{Variables}

$p$

$g$

h

$x$

$v^{i n j}, v^{w i t h}$

$f_{g}$

$f_{e}$

EENS

$C R$

\section{Sets}

I

$I_{N \& E}$
Outlet and inlet pressures of a gas compressor, respectively

Polytropic exponent of the empirical equation of gas compressors' consumed power

Horsepower of gas compressors

Natural gas thermal value

Natural gas emission factor

Efficiency of infrastructure

Electrical power consumed by a compressor

Gas consumed by a compressor

Total available budget in the base year

Allowed amount of not served energy

Value Of Lost Load

Duration of electrical, heat, and gas loads, respectively

The amount and duration of gas demand, respectively

Duration of total gas demand in equivalent gas and heat demand curve

Electricity and total gas demand (including gas demand of heat loads), respectively

\section{Generated power (MWh)}

Produced gas $\left(\mathrm{MM}^{3} / \mathrm{h}\right)$

Produced heat (MMBtu/h)

Binary decision variable

Injection and withdrawal of gas storage systems $\left(\mathrm{MM}^{3} / \mathrm{h}\right)$, respectively

Natural gas flow in a gas pipeline $\left(\mathrm{MM}^{3} / \mathrm{h}\right)$

Electric flow in a transmission line (MWh)

Expected Energy Not Served (MWh)

$$
\text { Curtailed electricity load (MW) }
$$

Set of candidate types corresponding to each infrastructure

Set of new-added and existent types of each infrastructure

\section{References}

1. Gillessen, B., Heinrichs, H., Hake, J.-F., et al. "Natural gas as a bridge to sustainability: Infrastructure expansion regarding energy security and system transition", Applied Energy, 251(1), pp. 113377-93 (2019). 
2. Pattupara. R. and Kannan, R. "Alternative lowcarbon electricity pathways in Switzerland and it's neighbouring countries under a nuclear phase-out scenario", Applied Energy, 172(3), pp. 152-68 (2016).

3. Sadeghi, H., Rashidinejad, M., Moeini-Aghtaie, A., et al. "The energy hub: An extensive survey on the stateof-the-art", Appl. Therm. Eng., 161(2), pp. 114071-97 (2019).

4. Yazdaninejadi, A., Hamidi, A., Golshannavaz, S., et al. "Impact of inverter-based DERs integration on protection, control, operation, and planning of electrical distribution grids", The Electr. J., 32(7), pp. 43-56 (2019).

5. Ahmadi, F., Akrami, A., Doostizadeh, M., et al. "Energy pricing and demand scheduling in retail market: how microgrids' integration affects the market", IET Smart Grid, 3(3), pp. 309-317 (2020).

6. Geidl, M., Koeppel, G., Favre-Perrod, P., et al. "The Energy Hub-A powerful concept for future energy systems", Third Annual Carnegie Mellon Conference on the Electricity Industry, pp. 14-19 (2007).

7. Nazar, M.S. and Haghifam, M.R. "Multiobjective electric distribution system expansion planning using hybrid energy hub concept", Electr. Power Syst. Res., 79(4), pp. 899-911 (2009).

8. Zhang, X., Shahidehpour, M., Alabdulwahab, A., et al. "Optimal expansion planning of energy hub with multiple energy infrastructures", IEEE Trans. on Smart Grid., 6(1), pp. 2302-11 (2015).

9. Tian, M.-W., Ebadi, A.G., Jermsittiparsert, K., et al. "Risk-based stochastic scheduling of energy hub system in the presence of heating network and thermal energy management", Appl. Therm. Eng., 159(3), pp. 113825-33 (2019).

10. Ghorab, M. "Energy hubs optimization for smart energy network system to minimize economic and environmental impact at Canadian community", Appl. Therm. Eng., 151(4), pp. 214-30 (2019).

11. Shanpour-Haghighi, A. and Seifi, A.R. "Effects of district heating networks on optimal energy flow of multicarrier systems", Renew. Sust. Energ. Rev., 59(7), pp. 379-87 (2016).

12. Rastegar, M., Fotuhi-Firuzabad, M., Zareipour, H., et al. "A probabilistic energy management scheme for renewable-based residential energy hubs", IEEE Trans. on Smart Grid, 8(1), pp. 2217-27 (2016).

13. Jing, R., Kuriyan, K., Kong, Q., et al. "Exploring the impact space of different technologies using a portfolio constraint based approach for multi-objective optimization of integrated urban energy systems", Renew. Sust. Energ. Rev., 113(2), p. 109249 (2019).

14. Unsihuay-Vila, C., Marangon-Lima, J., De Souza, A.Z., et al. "A model to long-term, multiarea, multistage, and integrated expansion planning of electricity and natural gas systems", IEEE Trans. on Power Syst., 25(1), pp. 1154-68 (2010).
15. Barati, F., Seifi, H., Sepasian, MS., et al. "Multi-period integrated framework of generation, transmission, and natural gas grid expansion planning for large-scale systems", IEEE Trans on Power Syst,, 30(5), pp. 2527-37 (2014).

16. Saldarriaga, C.A., Hincapié, R.A., and Salazar, H. "A holistic approach for planning natural gas and electricity distribution networks", IEEE Trans. on Power Syst., 28(1), pp. 4052-63 (2013).

17. Qiu, J., Dong, Z.Y., Zhao, J.H., et al. "Low carbon oriented expansion planning of integrated gas and power systems", IEEE Trans. on Power Syst., 30(6), pp. 1035-46 (2014).

18. Qiu, J., Yang, H., Dong, Z.Y., et al. "A linear programming approach to expansion co-planning in gas and electricity markets", IEEE Trans. on Power Syst., 31(3), pp. 3594-606 (2015).

19. Qiu, J., Dong, Z.Y., Zhao, J.H., et al. "Multi-stage flexible expansion co-planning under uncertainties in a combined electricity and gas market", IEEE Trans. on Power Syst., 30(4), pp. 2119-29 (2014).

20. Zhang, X., Shahidehpour, M., Alabdulwahab, A.S., et al. "Security-constrained co-optimization planning of electricity and natural gas transportation infrastructures", IEEE Trans. on Power Syst., 30(7), pp. 298493 (2014).

21. Hu, Y., Bie, Z., Ding, T., et al. "An NSGA-II based multi-objective optimization for combined gas and electricity network expansion planning", Applied Energy, 167(1), pp. 280-93 (2016).

22. Moeini-Aghtaie, M., Farzin, H., Fotuhi-Firuzabad, M., et al. "Generalized analytical approach to assess reliability of renewable-based energy hubs", IEEE Trans on Power Syst., 32(4), pp. 368-77 (2016).

23. Wang, X., Bie, Z., Liu, F., et al. "Bi-level planning for integrated electricity and natural gas systems with wind power and natural gas storage", Int. J. Electr. Power \& Energ. Syst., 118(1), p. 105738 (2020).

24. Liang, J. and Tang, W., "Stochastic multistage coplanning of integrated energy systems considering power-to-gas and the cap-and-trade market", Int. J. Electr. Power \& Energ. Syst., 119(1), p. 105817 (2020).

25. Odetayo, B., Kazemi, M., MacCormack, J., et al. "A chance constrained programming approach to the integrated planning of electric power generation, natural gas network and storage", IEEE Trans on Power Syst., 33(2), pp. 6683-99 (2018).

26. Pelletier, C. and Wortmann, J. "A risk analysis for gas transport network planning expansion under regulatory uncertainty in Western Europe", Energy Policy, 37(3), pp. 721-32 (2009).

27. Ciarreta, A., Espinosa, M.P., and Pizarro-Irizar, C. "Optimal regulation of renewable energy: A comparison of feed-in tariffs and tradable green certificates in the Spanish electricity system", Energy Econ, 67(1), pp. 387-99 (2017). 
28. Afful-Dadzie, A., Afful-Dadzie, E., Awudu, I., et al. "Power generation capacity planning under budget constraint in developing countries", Applied Energy, 188(1), pp. 71-82 (2017).

29. Iranian Power System Data, Aug. 2016 [Online]. Available:http://www.tavanir.org.ir

30. Sheikhhoseini, M., Rashidinejad, M., Ameri, M., et al. "Economic analysis of support policies for residential photovoltaic systems in Iran", Energy, 165(4), pp. 853-66 (2018).

31. Careri, F., Genesi, C., Marannino, P., et al. "Generation expansion planning in the age of green economy", IEEE Trans on Power Syst., 26(1), pp. 2214-23 (2011).

32. Dolatabadi, A. and Mohammadi-Ivatloo, B. "Stochastic risk-constrained scheduling of smart energy hub in the presence of wind power and demand response", Appl. Therm. Eng., 123(2), pp. 40-9 (2017).

33. Badami, M., Modica, S., and Portoraro, A. "A biofuelbased cogeneration plant in a natural gas expansion system: an energetic and economic assessment", Appl. Therm. Eng., 118(1), pp. 52-61 (2017).

\section{Biographies}

Hadi Sadeghi received his BSc and MSc degrees from South Tehran Branch, Islamic Azad University, Tehran, Iran and Shahid Bahonar University of Kerman, Kerman, Iran in Electrical Engineering in 2011 and 2014, respectively, where he is currently striving for the $\mathrm{PhD}$ degree. His research interests are in operation and expansion planning of energy systems, smart grids, and multi-carrier energy systems.
Masoud Rashidinejad received BSc degree in Electrical Engineering and MSc degree in Systems Engineering from Isfahan University of Technology, Isfahan, Iran, and the $\mathrm{PhD}$ degree in Electrical Engineering from Brunel University, London, U.K. in 2000. He is a Professor at the Department of the Electrical Engineering, Shahid Bahonar University of Kerman, Kerman, Iran. His research interests include power system optimization, power system planning, electricity restructuring, energy efficiency, and energy management in smart electricity grids.

Moein Moeini-Aghtaie received the MSc and PhD degrees from Sharif University of Technology, Tehran, Iran in 2010 and 2014, respectively, all in Electrical Engineering. He is currently an Assistant Professor at the Department of Electrical Engineering, Sharif University of Technology, Tehran, Iran. His current research interests include reliability and resilience studies of modern distribution systems, especially in the multicarrier energy environment, and charging management of plug-in hybrid electric-vehicles.

Amir Abdollahi received the BSc degree from Shahid Bahonar University of Kerman, Kerman, Iran in 2007, MSc degree from Sharif University of Technology, Tehran, Iran in 2009, and the PhD degree from Tarbiat Modares University, Tehran in 2012, all in in Electrical Engineering. Dr. Abdollahi is currently an Associate Professor at the Department of Electrical Engineering, Shahid Bahonar University of Kerman. His research interests include demand-side management, resiliency, reliability, and economics in smart electricity grids. 